\title{
Métodos de decisão multicritério para seleção de fornecedores: um panorama do estado da arte
}

\section{Multicriteria decision methods for supplier selection: a literature review on the state of the art}

\author{
Francisco Rodrigues Lima Junior ${ }^{1}$ \\ Lauro Osiro ${ }^{1}$ \\ Luiz Cesar Ribeiro Carpinetti ${ }^{1}$
}

\begin{abstract}
Resumo: A seleção de fornecedores se configura como uma das atividades mais críticas para a gestão de cadeias de suprimentos e influencia diretamente o desempenho das organizações, a qualidade dos produtos gerados, entre outros fatores de produção. Na literatura acadêmica, o processo de seleção de fornecedores tem sido abordado como um problema de decisão multicritério no qual requisitos da organização compradora devem ser convertidos em critérios para avaliar as alternativas de fornecimento. Para lidar com esse problema, diversos modelos teóricos vêm sendo propostos a partir de métodos de decisão multicritério, os quais costumam ser combinados com outras técnicas. Foram identificados cinco artigos de revisão da literatura sobre este tema, sendo que o trabalho mais recente analisou estudos publicados entre 2000 e 2008. Este artigo complementa as revisões anteriores analisando 120 artigos sobre o tema publicados em diversas bases de artigos, internacionais e nacionais. Com base nos artigos revisados, são discutidos os métodos de decisão multicritério mais utilizados, os contextos de aplicação, a origem dos estudos, entre outras informações relevantes. Os resultados mostraram que alguns métodos capazes de lidar com incertezas e informações qualitativas são os mais utilizados e que a maioria dos modelos parecem não expressar as reais necessidades das empresas. Ao final, são identificadas algumas lacunas para realização de pesquisas futuras.

Palavras-chave: Métodos de decisão multicritério (MCDM). Seleção de fornecedores. Gestão da cadeia de suprimentos.
\end{abstract}

\begin{abstract}
Supplier selection is a very important activity in supply chain management, and it directly influences the performance of organizations, product quality, and other factors related to the production process. Supplier selection has been addressed as a multicriteria decision problem that considers that the requirements of the buyers should focus on criteria used to evaluate the supplier options available. Various multicriteria decision methods or a combination of them have been proposed to tackle this problem. Three articles were identified during a literature review on this topic. The most recent article cited studies from 2000 to 2008. This paper also includes the review of other 120 articles that were retrieved from the literature search using different national and international data bases. Based on the articles reviewed, the most commonly used multicriteria decision methods, context of applications, study sources, among other relevant information. The results show that methods capable of dealing with uncertainty and qualitative information are the most common. Also, it seems that there is a gap between real world needs and the proposed theoretical models. Finally, the present study identifies areas that need further research.
\end{abstract}

Keywords: Multicriteria decision methods (MCDM). Supplier selection. Supply chain management.

\section{Introdução}

Mediante as configurações dos sistemas produtivos emergentes, os quais podem ser caracterizados por cadeias ou redes de suprimentos, pelo uso de tecnologias que ampliam as possibilidades de compra e por mudanças constantes nas necessidades dos clientes, o processo de seleção de fornecedores em organizações industriais tem se tornado cada vez mais importante e complexo (DE BOER; WEGER; TELGEN, 1998; DE BOER; PIERANGELA; MORLACCHI, 2001; PIDDUCK, 2006; CASTRO; GOMEZ; FRANCO, 2009).
A seleção de fornecedores se configura como uma das atividades mais críticas para o estabelecimento de uma cadeia de suprimentos efetiva (CHENG; LIN; HUANG, 2006), afetando o desempenho das organizações (THRULOGACHANTAR; ZAILANI, 2011), a flexibilidade do sistema de manufatura (NDUBISI et al., 2005), o resultado da implementação de programas de qualidade e de melhoria contínua e, consequentemente, a qualidade dos produtos gerados e o atendimento de requisitos dos clientes finais

\footnotetext{
Escola de Engenharia de São Carlos, Universidade de São Paulo - USP, São Carlos, SP, Brasil, e-mail: franciscojr@sc.usp.br; lauro.osiro@gmail.com; carpinet@sc.usp.br
} 
(GONZÁLEZ et al., 2004; WANG, 2010). Neste sentido, as estratégias de compra devem buscar o alinhamento dos objetivos de compra com os objetivos da organização, exigindo um papel mais pró-ativo por parte da empresa na seleção de fornecedores e sua contínua avaliação (DE BOER; PIERANGELA; MORLACCHI, 2001; PRESSEY; WINKLHOFER; TZOKAS, 2009).

Nesse contexto, por depender de inúmeros fatores, o processo de seleção de fornecedores vem sendo predominantemente abordado na literatura científica como um problema de decisão no qual diversos critérios de decisão podem ser considerados no julgamento das possíveis empresas fornecedoras. Outro aspecto complicador do processo de decisão para seleção de fornecedores é a natureza subjetiva dos critérios de seleção e também da importância relativa desses critérios no processo de tomada de decisão para seleção (DE BOER; PIERANGELA; MORLACCHI, 2001; HA; KRISHNAN, 2008; WANG, 2010).

Para lidar com tal problema, métodos de decisão multicritério (métodos MCDM - Multi Criteria Decision Making Methods) ou ainda métodos estatísticos, programação matemática e de inteligência artificial (IA) (HA; KRISHNAN, 2008; HO; XU; DEY, 2010) vêm sendo explorados. Em buscas às bases de dados, foram localizados cinco artigos de revisão da literatura sobre a utilização de métodos MCDM no processo de seleção de fornecedores (WEBER; CURRENT; BENTON, 1991; DEGRAEVE; LABRO; ROODHOOFT, 2000; DE BOER; PIERANGELA; MORLACCHI, 2001; AISSAOUI; HAOUARI; HASSINI, 2007; HO; XU; DEY, 2010), sendo o trabalho mais recente datado de 2010, no qual os autores analisaram trabalhos publicados entre 2000 e 2008. No entanto, há grande emergência de estudos sobre este assunto mediante o foco que a gestão da cadeia de suprimentos e as relações interempresariais têm recebido de pesquisas em gestão de operações (CHENG; LIN; HUANG, 2006; PIDDUCK, 2006; AMBROSE et al., 2008). Desta forma, o trabalho apresentado neste artigo procura contribuir na direção de atualizar o mapeamento do estado da arte e apontar lacunas deste tema de pesquisa.

Segundo Kitchenham e Charters (2007), a menos que uma revisão da literatura seja completa e justa, é de pouco valor científico, o que justifica também a necessidade de realizar revisões da literatura de forma sistematizada. Diante do exposto, este estudo tem o objetivo de traçar um panorama da literatura científica que contemple o desenvolvimento e/ou aplicação de métodos de decisão multicritério para o processo de seleção de fornecedores por meio de um levantamento bibliográfico sistematizado, visando, assim, contribuir para o desenvolvimento deste tema de pesquisa na comunidade científica. Foram analisados 120 artigos sobre o tema no intuito de investigar quais os métodos MCDM e técnicas diversas são os mais utilizados na elaboração de modelos de decisão, a origem dos estudos, as aplicações para validação dos modelos, entre outras informações relevantes.

Quanto à estrutura do artigo, a Seção 2 consiste em uma revisão bibliográfica que contempla aspectos relacionados à seleção de fornecedores e à modelagem multicritério. Em seguida, a Seção 3 descreve os procedimentos de pesquisa utilizados neste estudo e a Seção 4 apresenta e discute os resultados desta pesquisa. Por fim, a Seção 5 apresenta algumas considerações finais, destacando algumas oportunidades de pesquisa sobre o tema.

\section{Seleção de fornecedores}

\subsection{O Processo de seleção de fornecedores}

$\mathrm{Na}$ literatura de pesquisa operacional, a seleção de fornecedores tem sido abordada como um problema de decisão multicritério cujo objetivo principal consiste em encontrar os fornecedores certos que estejam aptos a oferecer à empresa compradora produtos ou serviços com a qualidade desejada, com preço justo, no tempo certo e na quantidade especificada (HA; KRISHNAN, 2008; BORAN et al., 2009). De forma mais abrangente e sob uma visão processual, De Boer, Labro e Morlacchi (2001) e Aissaoui, Haouari e Hassin (2007) estruturam o processo de seleção de fornecedores em 4 etapas inter-relacionadas que visam identificar um conjunto de possíveis fornecedores e reduzi-lo até a escolha final, sendo estas:

- Definição do problema: O processo se inicia com um questionamento sobre o que exatamente se pretende alcançar por meio da seleção de fornecedores. De um lado, existem empresas que frequentemente buscam novos fornecedores para atualizar seu mix de produtos quanto à variedade e tipologia. Em outra via, há empresas situadas em ambientes que envolvem relacionamentos próximos com fornecedores, desenvolvendo parcerias e contratos de longo prazo. Desta forma, o agente decisório se depara com diferentes situações que devem conduzir a diferentes decisões. Para fazer a escolha certa, o processo de compras deve inicialmente definir claramente quais os objetivos do processo de seleção;

- Formulação dos critérios: Nesta etapa, a organização compradora deve focar esforços no estabelecimento de critérios de decisão que exprimam claramente os seus requisitos, desde o nível operacional ao nível estratégico. De acordo com Kahraman, Cebeci e Ulukan (2003), 
pode não ser fácil converter as necessidades em critérios utilizáveis, já que estas geralmente são expressas como conceitos qualitativos;

- Qualificação: A qualificação consiste em reduzir o conjunto de todos fornecedores para um conjunto que possa ser avaliado mais detalhadamente durante a escolha final. A qualificação pode ser conduzida em um ou vários passos, nos quais diferentes métodos de eliminação podem ser aplicados caso uma alternativa não satisfaça alguma regra de seleção.

- Escolha final: Nesta última etapa, deve-se ordenar os fornecedores qualificados e pode-se considerar a seleção de um ou mais fornecedores. Se mais de um fornecedor for selecionado, esta etapa também envolve a alocação de pedidos entre os selecionados, a qual deve considerar a possibilidade de alocar um fornecedor para um produto ou para um grupo de itens de uma só vez.

Castro, Gomez e Franco (2009) apontaram os principais aspectos que influenciam a elaboração de estratégias e o processo decisório para seleção de fornecedores. Os autores destacam que a seleção de fornecedores pode ser bastante difícil devido à natureza e à diversidade dos produtos e serviços adquiridos e às variações quantitativas e qualitativas no comportamento da demanda. Os autores atentam para a necessidade de realizar uma análise contextual que deve considerar aspectos como o tamanho desejado para a base de fornecedores (abastecimento com fonte única ou abastecimento com fonte múltipla), a relação com os fornecedores (simples transações comerciais ou parcerias estratégicas de longo prazo) e as possíveis situações de compra (compra pela primeira vez, recompra modificada e recompra para produtos de rotina). Estes aspectos ajudam a determinar o desdobramento de cada uma das fases do processo de seleção e, consequentemente, influenciam a complexidade e a importância da decisão. Diante disso, modelos de decisão específicos para apoiar cada etapa do processo de seleção de fornecedores em cada uma das situações de compra vêm sendo propostos na literatura.

Diante de tal importância e complexidade, o processo de seleção de fornecedores tem obtido bastante atenção no atual ambiente de negócios, dadas as incertezas inerentes às decisões de compras e a possibilidade de obter ganhos decorrentes da cooperação interempresarial. Para obter uma decisão final efetiva neste domínio de problema, é importante escolher o(s) método(s) e os critérios de decisão que melhor condizem com as peculiaridades do problema e com a realidade da empresa.

\subsection{Critérios de decisão para seleção de fornecedores}

Os critérios de decisão para seleção de fornecedores são atributos qualitativos ou quantitativos utilizados na avaliação das diferentes possibilidades de fornecedores. A literatura relacionada à seleção de fornecedores identifica dezenas de critérios de natureza quantitativa e qualitativa que podem ser considerados durante a seleção de fornecedores. Kahraman, Cebeci e Ulukan (2003) propõem uma categorização dos critérios para seleção de fornecedores que os subdivide em critérios relativos à: reputação dos fornecedores, desempenho de produtos fornecidos, desempenho de serviços e, por último, desempenho associado a custos.

Dickson (1966) realizou um estudo pioneiro no mapeamento desses critérios, o qual envolveu a participação de 170 profissionais de compras. Os resultados mostraram que "qualidade", "entrega", "histórico de desempenho" e "garantia" foram considerados os critérios de maior relevância pelos entrevistados. Alguns anos depois, Weber, Current e Benton (1991) realizaram uma pesquisa utilizando 74 artigos publicados após o trabalho de Dickson (1966) e constataram que os critérios que mais receberam atenção entre os estudos selecionados são "custo", "qualidade" e "entrega”. Já o estudo de Frödell (2011) contou com a participação de 12 profissionais de compras e constatou que os critérios que costumam receber mais atenção estão associados primeiramente ao "custo", seguidos por "competências-chave", "complacência" e "capacidade para colaboração e desenvolvimento", apontando uma tendência para o aumento da importância de critérios relacionados a parcerias e colaboração em redes de suprimentos.

Conforme mencionado anteriormente, os critérios podem possuir natureza quantitativa, tais como custo e prazo de entrega, ou qualitativa, como comunicação com o fornecedor, confiança e capacidade de P\&D. É interessante observar que, mesmo para critérios de natureza quantitativa, na fase de seleção de fornecedores, ainda que o fornecedor em questão já tenha fornecido para a empresa compradora, a avaliação sobre qual será seu desempenho futuro é, na melhor das hipóteses, uma boa estimativa. Nesse contexto, diferentes métodos de decisão podem ser adotados, considerando os diversos aspectos de adequação de um ou mais métodos para decisões em seleção de fornecedores.

\subsection{Métodos de decisão multicritério para seleção de fornecedores}

A modelagem de um problema de decisão pode contar com a participação de um ou mais agentes decisórios e utilizar um ou mais critérios durante a avaliação. Cada agente decisório é responsável por definir valores de julgamento pessoal a alguns 
atributos, tais como o grau de desempenho das alternativas em relação a cada critério e o peso (ou nível de pertinência) dos critérios de decisão. Desta forma, os valores dessas variáveis são influenciados por fatores subjetivos decorrentes da intuição e da experiência dos agentes decisórios (KLIR, 1995; GOMES, 2004; KAHRAMAN, 2008).

Os métodos de decisão que consideram mais de um critério são definidos como métodos de decisão multicritério (métodos MCDM). Nesses métodos, algumas alternativas são avaliadas segundo um número de critérios definidos, sendo que cada critério induz a uma ordenação particular das alternativas, fazendo com que seja necessária a adoção de algum mecanismo capaz de construir uma ordenação geral de preferências, também chamada de ranking ou classificação (KAHRAMAN, 2008).

De Boer, Wegen e Telgen (1998) destacam que os métodos de decisão multicritério para seleção de fornecedores diferem a partir da definição do número de critérios e sua natureza; das situações de decisão possíveis, do número de agentes decisórios, da inter-relação das decisões; e do tipo de regra de decisão a ser usada (compensatórias ou não compensatórias).

A literatura sob o tema identifica três situações de tomada de decisão com as quais esses métodos devem ser capazes de lidar. As decisões sob riscos (incertezas estocásticas) são caracterizadas pela falta de certeza sobre os acontecimentos futuros, o que é recorrente em situações de seleção de fornecedores devido a flutuações de mercado, ou seja, há mais de um estado de natureza e pressupõe-se que o(s) agente(s) decisório(s) seja(m) capaz(es) de mensurar uma estimativa de probabilidade para ocorrência de cada um dos estados. As decisões sob incerteza são caracterizadas pelos fenômenos de imprecisão e indeterminação presente nelas, o que também é bastante comum no processo de tomada de decisão em seleção de fornecedores. A imprecisão se refere à dificuldade de determinar os pesos dos critérios e das pontuações das alternativas, mesmo não existindo a possibilidade de flutuação aleatória destes valores. Já o fenômeno de indeterminação consiste na interpretação ambígua dos critérios de decisão e faz com que o agente decisório não saiba exatamente o que deve ser medido (por exemplo, capacidade de P\&D). Por fim, e menos comum em situações de seleção de fornecedores, as decisões sob certeza são aquelas em que se sabe qual(is) estado(s) de natureza acontecerá(ão) (DE BOER; WEGEN; TELGEN, 1998; ANDRADE, 1998; MOORE, 2005; CHENG; LIN; HUANG, 2006).

Vários autores categorizam os métodos MCDM segundo duas abordagens: Multiple Attribute Decision Making (MADM) e Multiple Objective Decision Making (MODM) (BHUTTA; HUQ, 2002; FIGUEIRA; GRECO; EHRGOT, 2005; KAHRAMAN,
2008). A abordagem MADM consiste em fazer seleção entre alternativas predeterminadas na presença de múltiplos atributos, os quais são geralmente conflitantes, sendo que a quantidade de alternativas deve ser limitada e as alternativas descritas por meio de seus atributos relacionados. A partir disso, é possível desenvolver um processo de classificação das alternativas (KAHRAMAN, 2008).

Embora a abordagem MADM propicie soluções mais factíveis e passíveis de implementação, há situações em que é necessário modelar o problema de seleção de fornecedores por meio da abordagem MODM. A principal justificativa para adoção da abordagem MODM está no fato de que a decisão final desse problema influencia diferentes funções dentro de uma organização (tais como compras, qualidade, produção, entre outras) e, portanto, a organização deve buscar maximizar o desempenho de cada uma destas funções por meio da obtenção de resultados "ótimos" na decisão. Uma vantagem dessa abordagem é a possibilidade de permitir a alocação de diferentes volumes de aquisição para cada fornecedor. Contudo, este é um processo complexo e, em muitos casos, é impossível implementar a solução na prática (BHUTTA; HUQ, 2002).

A resolução de um modelo MCDM deve obedecer a um conjunto de regras determinadas pelo(s) método(s) escolhido(s) para abordar o problema, sendo que esta escolha deve ser feita ainda na fase de desenvolvimento do modelo. De Boer, Weger, Telgen (1998) destacam que os métodos MCDM são capazes de contribuir para a eficiência da tomada de decisão por justificar os processos de decisão, permitir o processamento mais rápido e automatizado dos dados, bem como o armazenamento dos processos de decisão, possibilitando assim o acesso à informação em situações futuras. Wang (2010) acrescenta que esses métodos são importantes por abordar difíceis decisões em organizações, já que auxiliam os gestores em situações de incerteza, complexidade e objetivos conflitantes.

A literatura identifica diversos métodos MCDM que podem ser utilizados em modelos simples ou modelos combinados. Entre esses, destacam-se os métodos Análise por Envoltório de Dados (Data Envelopment Analysis - DEA) (CHARNES; COOPER; RHODES, 1978), Método Analítico Hierárquico (Analytic Hierarchy Process - AHP) (SAATY, 1980), Método Analítico de Redes (Analytic Network Process - ANP) (SAATY, 1996), métodos diversos de programação matemática, além de abordagens baseadas em técnicas computacionais e de IA, como Fuzzy (ZADEH, 1965), Redes Neurais Artificiais (RNA), Algoritmo Genético (HOLLAND, 1992) e várias outras que são resultado da combinação de duas ou mais técnicas. De modo geral, tais métodos diferem por suas limitações, 
adequação ao contexto de uso e forma de abordagem das peculiaridades do problema (HO; XU; DEY, 2010).

Uma visão geral sobre a evolução do uso de métodos de decisão para seleção de fornecedores é apresentada na Figura 1, destacando alguns marcos em uma escala temporal. Esses marcos incluem a proposição de alguns métodos MCDM e de sobreclassificação (outranking), técnicas de gestão e de IA, assim como a proposição de modelos de decisão pioneiros na utilização de determinados métodos e técnicas. A Figura 1 aponta também alguns eventos que contribuíram e influenciaram na evolução dos conceitos e técnicas de seleção de fornecedores.

Há várias perspectivas para classificação dos métodos MCDM para seleção de fornecedores. Ha e Krishnan (2008) os classificam em métodos matemáticos, estatísticos e de IA. Os métodos matemáticos apresentam melhor desempenho em situações de certeza, já que não são capazes de considerar riscos, imprecisão e subjetividade. Em outra via, os métodos estatísticos estão voltados para situações de incertezas estocásticas, nas quais deve ser possível determinar probabilidades de ocorrência de determinados eventos.

De Boer, Pierangela e Morlacchi (2001) afirmam que a maioria dos métodos utilizados para seleção e avaliação de fornecedores considera somente critérios quantitativos e isso pode afetar negativamente a composição da base de fornecedores. Chou, Shen e Chang (2007) alertam que a imprecisão é inerente à natureza de algumas informações relevantes no problema de seleção de fornecedores, tais como critérios de decisão qualitativos avaliados por meio da percepção humana e de julgamento, os quais não podem ser quantificados precisamente. Portanto, nota-se que abordagens que empregam somente números exatos não são capazes de apoiar efetivamente o processo para seleção de fornecedores. Neste sentido, algumas técnicas de IA teoricamente apresentam

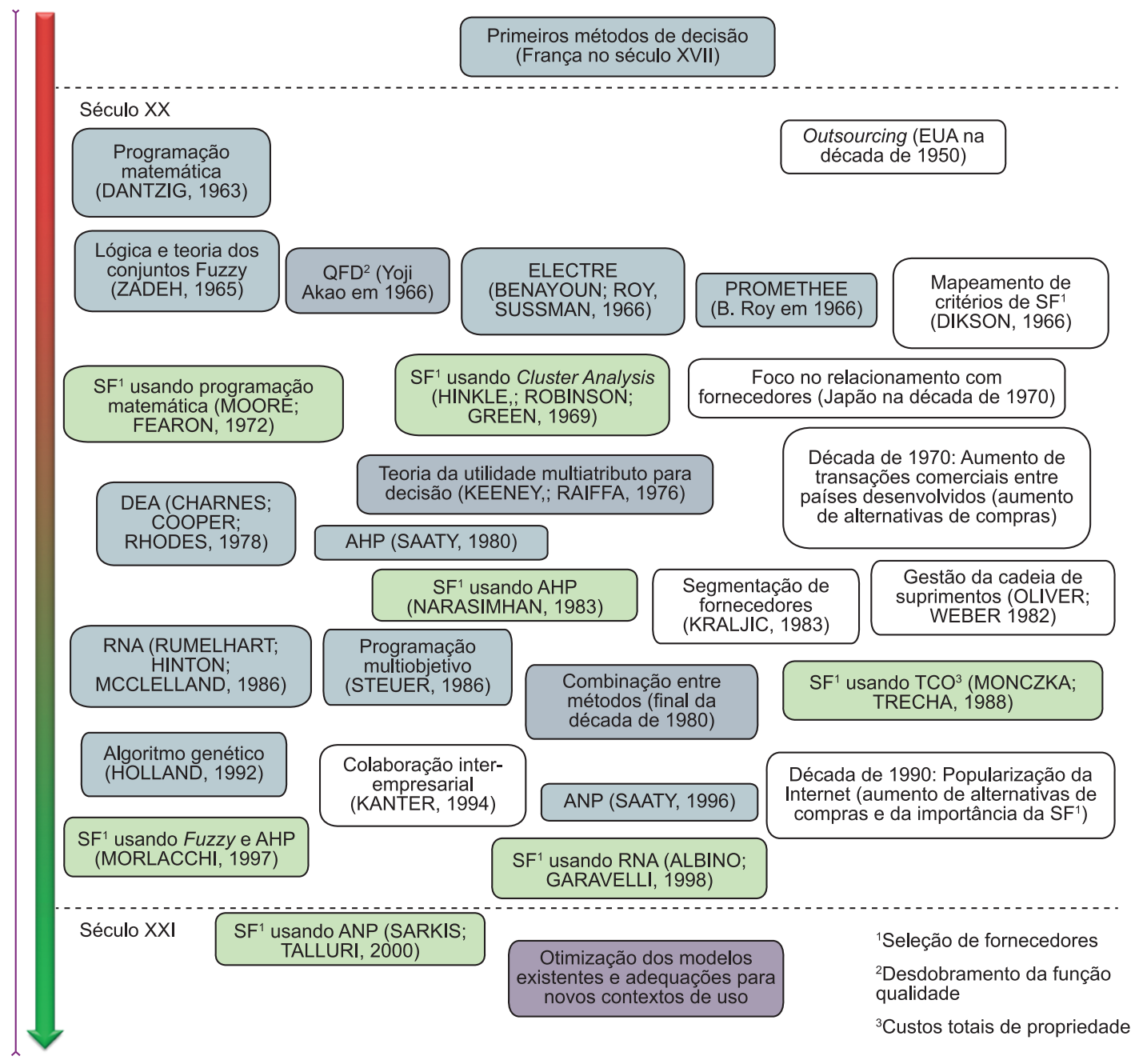

Figura 1. Alguns marcos do uso de métodos de decisão para seleção de fornecedores. 
melhor desempenho por serem capazes de lidar com fenômenos de incerteza.

Diante de várias situações e necessidades, o levantamento bibliográfico relatado neste artigo identificou diversas abordagens propostas para o problema de seleção de fornecedores. A Seção 3, a seguir, apresenta os procedimentos adotados para selecionar e analisar diversos artigos que propõem o desenvolvimento e/ou aplicação de modelos MCDM.

\section{Procedimento de pesquisa bibliográfica}

O método de pesquisa utilizado foi desenvolvido a partir de procedimentos recomendados para elaboração de revisões sistemáticas. Kitchenham e Charters (2007) definem "revisão sistemática da literatura" como um meio de identificar, avaliar e interpretar todas as pesquisas disponíveis relevantes para uma questão de pesquisa específica, ou área temática, ou fenômeno de interesse. Sampaio e Mancini (2007) acrescentam que a elaboração de uma revisão sistemática deve identificar as bases de dados a serem consultadas, definir palavras-chave e estratégias de busca, estabelecer critérios para a seleção de artigos, comparar as buscas dos examinadores, definir a seleção inicial de artigos, aplicar os critérios na seleção destes e justificar possíveis exclusões. Além disso, deve-se analisar criticamente e avaliar todos os estudos incluídos na revisão, preparar um resumo crítico, sintetizando as informações disponibilizadas pelos artigos que foram incluídos na revisão e apresentar uma conclusão.

Neste sentido, a pesquisa bibliográfica realizada obedeceu a tais recomendações. Os artigos selecionados foram coletados nos portais de periódicos Science Direct (www.sciencedirect.com), Web of Science (apps.webofknowledge.com), Scopus (www. scopus.com) e Emerald (www.emeraldinsight.com) de forma sistematizada. Para coletar artigos nos portais de periódicos, foram definidos os seguintes procedimentos de busca e seleção: (1) Inserção da palavra-chave "supplier selection and evaluation problem" nos respectivos campos de busca em cada um dos portais; (2) Utilização de filtros para selecionar somente artigos publicados entre 2002 e 2011 e que foram publicados em periódicos científicos (Journals); (3) Ordenação dos resultados por "relevância" a partir de uma funcionalidade contida em cada um dos portais; (4) Seleção dos 100 primeiros resultados listados; (5) Eliminação de artigos que não contemplavam o desenvolvimento e/ou aplicação de modelos de decisão para o problema de seleção de fornecedores; e (6) Eliminação de cópias dos artigos repetidos, ou seja, aqueles que foram listados e selecionados em mais de um portal. O Quadro 1 apresenta a quantidade de artigos resultante após a execução de cada um dos procedimentos de coleta descritos.

As buscas restringiram os artigos publicados nos últimos 10 anos (2002 a 2011) para obter uma gama de artigos mais representativa do panorama atual deste tema de pesquisa. Artigos publicados até 2002 foram analisados nas revisões sistemáticas anteriores, feitas por De Boer, Pierangela e Morlacchi (2001), Degraeve, Labroe e Roodhooft (2000) e Weber, Current e Benton (1991).

Alguns imprevistos ocorreram durante a realização da busca e seleção de artigos. Durante a busca no Emerald, a palavra-chave foi substituída por "supplier selection", já que todos os artigos coletados foram eliminados durante o procedimento 5 por estarem fora do escopo desta pesquisa. No caso da busca no Scopus, como não apresenta a funcionalidade de buscar somente artigos de periódicos, entre os 193 resultados listados somente 25 artigos foram coletados, já que os demais consistiam em trabalhos publicados em anais de eventos ou somente resumos de artigos de periódicos.

Quanto aos artigos eliminados, estes abordavam assuntos como ferramenta de benchmarking para seleção de fornecedores, critérios para seleção de fornecedores, revisão bibliográfica sobre métodos de decisão, gestão da cadeia de suprimentos, gestão de serviços, comparação entre métodos MCDM e revisões da literatura sobre métodos de decisão existentes para seleção de fornecedores. Portanto, foram analisados 120 artigos, dos quais, foram extraídas informações como método(s) utilizado(s) no desenvolvimento do modelo, setor econômico da empresa em que foi aplicado, periódico e país de origem, critérios utilizados, entre outras informações pertinentes. Os dados foram organizados em uma planilha eletrônica e os resultados são apresentados e discutidos na Seção 4.

Quadro 1. Quantidade de artigos resultante de cada um dos procedimentos de coleta.

\begin{tabular}{lcccccc}
\hline \multirow{2}{*}{ Portais de periódicos } & \multicolumn{7}{c}{ Etapas de busca e seleção } \\
\cline { 2 - 7 } & $\mathbf{1}$ & $\mathbf{2}$ & $\mathbf{3}$ & $\mathbf{4}$ & $\mathbf{5}$ & $\mathbf{6}$ \\
\hline Science Direct & 18.693 & 8.878 & 8.878 & 100 & 85 & 85 \\
Emerald & 2869 & 1581 & 1581 & 100 & 22 & 18 \\
Web of Science & 102 & 46 & 46 & 40 & 25 & 13 \\
Scopus & 208 & 193 & 193 & 25 & 19 & 4 \\
Total de artigos selecionados & - & - & - & - & 147 & 120 \\
\hline
\end{tabular}




\section{Apresentação e discussão dos resultados}

Esta seção está focada na caracterização de pesquisas selecionadas, não sendo objetivo realizar revisão bibliográfica sobre especificidades dos métodos identificados, mediante a grande quantidade de métodos e a limitação de tamanho deste artigo.

Embora o total de estudos analisados (referenciados também como "trabalhos", "pesquisas" ou "artigos") nesta pesquisa seja de 120, o somatório das quantidades de determinadas ocorrências de eventos na amostra utilizada (dispostas em gráficos) pode ultrapassar este número. Isso se deve ao fato de que, por exemplo, um estudo pode ser originário de mais de um país, assim como pode abordar a utilização de mais de um método.

\subsection{Proveniência dos estudos analisados}

A Figura 2 apresenta a quantidade de estudos selecionados segundo o ano de publicação. Conforme citado anteriormente, esta pesquisa contempla artigos publicados entre 2002 e 2011. No entanto, de acordo com Figura 2, a maioria dos trabalhos selecionados para compor este estudo se concentra em estudos publicados recentemente. Entre os 120 artigos selecionados, $73(60,8 \%)$ foram publicados de 2009 até 2011, ou seja, estes não foram considerados nas pesquisas existentes de revisão da literatura. Acredita-se que a predominância de artigos mais recentes, também considerados mais "relevantes" pelos portais de periódicos, fortaleça o esforço desta seção em caracterizar as tendências de pesquisas no que se refere à identificação de métodos mais recentemente utilizados e tipos de aplicação.

Quanto à procedência das pesquisas, a Figura 3 apresenta a quantidade de estudos selecionados segundo o país de origem.

De acordo com os artigos levantados nesta pesquisa, nota-se a predominância de estudos oriundos de Taiwan (22,5\%), Turquia (20,83\%), Irã (17,5\%) e China (7,5\%), sendo que somente estes quatro países são responsáveis pelo desenvolvimento de $68,33 \%$ das pesquisas. Uma constatação interessante é que nenhum estudo de origem brasileira foi encontrado nessa amostra, o que parece mostrar que este assunto tem sido pouco discutido no Brasil ou que os estudos produzidos não vêm atingindo certo nível de relevância para a comunidade científica.

Quanto à identificação de periódicos que contemplem este tema de pesquisa, a Figura 4 mostra a quantidade de estudos selecionados segundo o periódico de origem.

A Figura 4 identifica os periódicos (Journals) nos quais os artigos foram publicados ou, para alguns casos, aceitos para publicação e, no momento em que foram coletados, encontravam-se em estado de espera para impressão. O periódico Expert Systems with Applications apresenta maior quantidade de estudos publicados sobre o tema de pesquisa $(31,66 \%)$, seguido do International Journal Production Economics, com

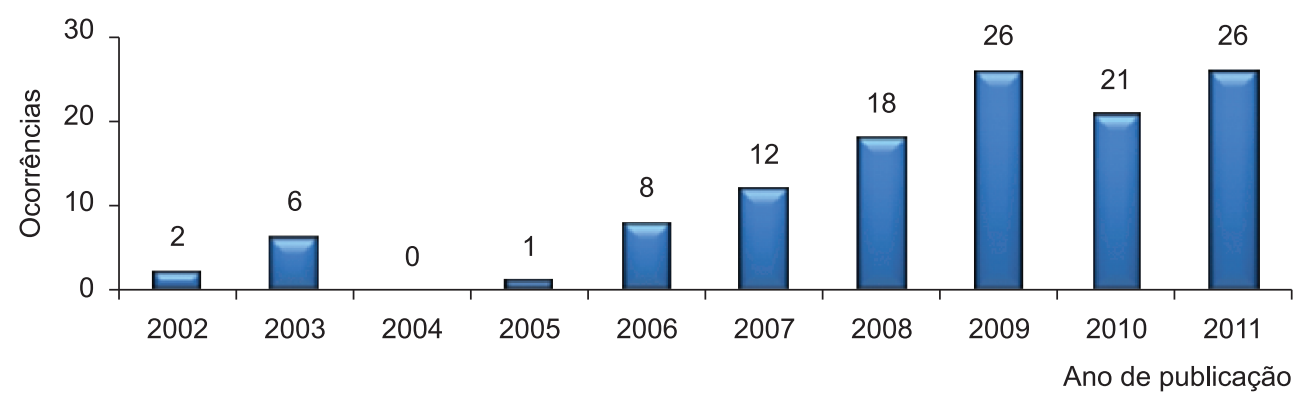

Figura 2. Quantidade de estudos por ano de publicação.

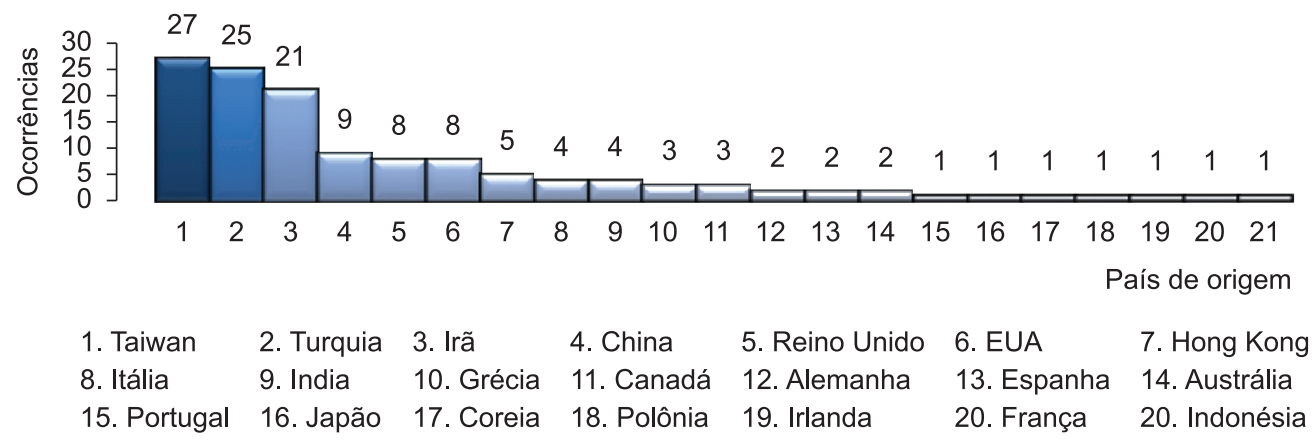

Figura 3. Quantidade de estudos por país de origem. 
9,16\% do total de pesquisas selecionadas. Embora exista vasta diversidade de periódicos que contemplem estudos sobre o tema, nota-se que predominantemente os dois periódicos mencionados parecem estar mais direcionados a abordar este tipo de estudo que os demais. Estas informações podem ser úteis aos pesquisadores deste tema que, ao selecionarem para qual periódico submeter seus respectivos artigos, terão a oportunidade de obter o crivo de avaliadores especializados no tema, o que tende a agregar valor ao estudo por meio de contribuições mais precisas e consistentes.

\subsection{Métodos MCDM, critérios de decisão e aplicações}

Nesta seção, são apresentados os resultados quantitativos referentes à ocorrência de uso de cada um dos métodos aplicados à seleção de fornecedores. Algumas técnicas apresentadas não são necessariamente métodos MCDM e, por isso, estes são combinados com outros métodos e/ou com técnicas de diversos tipos para definição dos critérios e modelagem do problema. Essa ressalva é válida para Redes Neurais Artificiais (RNA), Algoritmo Genético, abordagens fuzzy, Mineração de Dados, Raciocínio Baseado em Casos, QFD, SWOT, entre vários outros. Apesar disso, todas as técnicas serão referenciadas como métodos MCDM pela finalidade que lhes é atribuída nos modelos analisados e por questões de simplificação. A Figura 5 apresenta a quantidade de ocorrências de cada um dos métodos
MCDM simples, ou seja, métodos que não são mesclados com outro método.

De acordo com a Figura 5, as abordagens baseadas na teoria dos conjuntos fuzzy compõem o método MCDM mais utilizado de forma isolada, totalizando 8 ocorrências (CHEN; LIN; HUANG, 2006; ORDOOBADI, 2008; SHEN; YU, 2009; SHU; WU, 2009; ZHANG, 2009; HSU; CHIANG; SHU, 2010; LAM; TAO, 2010; WANG, 2010). Estas abordagens fuzzy realizam o ranqueamento de fornecedores por meio do uso de equações matemáticas desenvolvidas, do uso de operadores fuzzy ou por mecanismos de inferência.

Em segundo lugar, com 4 ocorrências cada um, encontram-se os métodos ANP (GENCER; GÜRPINAR， 2007; KIRYTOPOULOS; LEOPOULOS; VOULGARIDOU, 2008; HSU; HU, 2009; LEE; CHANG; LIN, 2009) e Algoritmo Genético (LIAO; RITTSCHER, 2007; CHE; WANG, 2008; REZAEI; DAVOODI, 2008; DAS; ROY; KAR, 2010). Com 3 ocorrências, o método DEA (SAEN, 2007a; SAEN, 2010; WU, 2010). Posteriormente, com 2 ocorrências cada um, situa-se o AHP (HOU; SU, 2006; SAEN, 2007b), TCO (MICHELI, 2008; VANTEDDU; CHINNAM; GUSHIKIN, 2011), Programação Inteira Mista (SAWIK, 2011; ZHANG; ZHANG, 2011) e PROMETHEE (DUMMING; MINNINO, 2003; ARAZ; OZKARAHAN, 2007). Por último, com 1 ocorrência cada um, encontram-se os métodos Programação Multiobjetivo (XIA; WU, 2007), Taguchi loss function (ORDOOBADI, 2009),

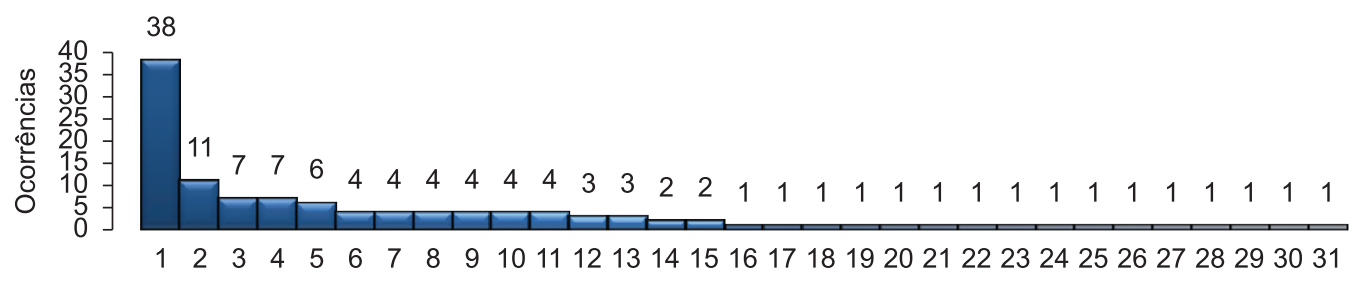

Periódicos

1. Expert Systems with Applications

3. Applied Mathematical Modelling

5. Supply Chain Management

7. European Journal of Operational Research

9. Information Sciences

11. Omega

13. Mathematical and Computer Modelling

15. Journal of Cleaner Production

17. Applied Mathematics and Computation

19. Benchmarking: An International Journal

21. Computers \& Operations Research

23. International Journal of Computer Integrated

Manufacturing

25. Industrial Management \& Data Systems

27. Journal of Enterprise Information

28. Journal of Manufacturing Technology Management

31. Measuring Business Excellence

Figura 4. Quantidade de estudos por periódico de origem.
2. International Journal Production Economics

4. Computers \& Industrial Engineering

6. Logistics Information Management

8. International Journal Advanced Manufacture Technology

10. Journal of Purchasing \& Supply Management

12. Computers in Industry

14. Applied Soft Computing

16. Advances in Engineering Software

18. Automation in Construction

20. Computers and Mathematics with Applications

22. International Journal of Physical Distribution \&

Logistics Management

24. International Journal of Production Research

26. Journal of Modelling in Management

29. Journal of the Franklin Institute

30. Management Decision . 


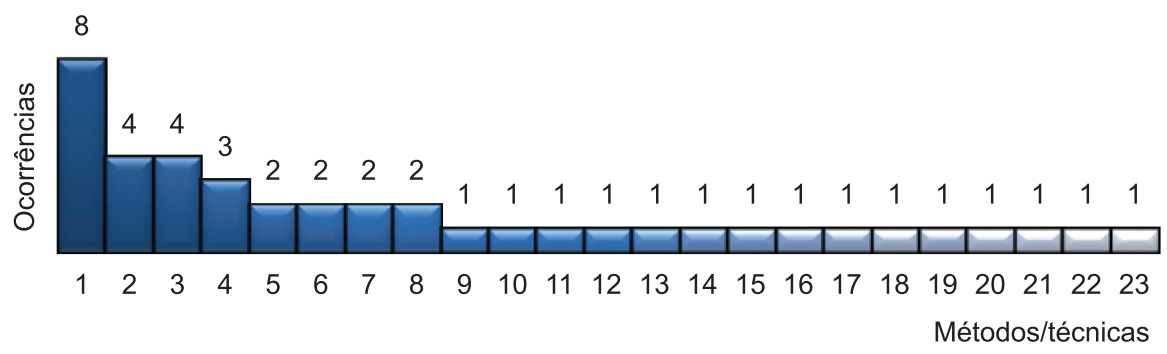

$\begin{array}{lll}\text { 1. Abordagem Fuzzy } & \text { 2. ANP } & \text { 3. Algoritmo genético } \\ \text { 4. DEA } & \text { 5. AHP } & \text { 6. PROMETHEE } \\ \text { 7. TCO } & \text { 8. Programação inteira mista } & \text { 9. Raciocínio baseado em casos } \\ \text { 10. Mineração de dados } & \text { 11. Grey-based Approach } & \text { 12. Power factors aggregation method } \\ \text { 13. Programação multiobjetivo } & \text { 14. Rough Set Theory } & \text { 15. Support vector machine } \\ \text { 16. Taguchi loss function } & \text { 17. Análise multivariada } & \text { 18. Redes neurais artificiais (RNA) } \\ \text { 19. Ant colony system } & \text { 20. Programação por metas } & \text { 21. MACBETH } \\ \text { 22. Modelo puramente matemático } & \text { 23. Programação linear } & \end{array}$

Figura 5. Métodos MCDM por quantidade de ocorrências.

Support Vector Machine (GUO; YUAN; TIAN, 2009), Rough Set Theory (CHANG; HUNG, 2010), Power Factors Aggregation Method (ZOLGHADRI, 2011), Grey-based Approach (LI; YAMAGUCHI; NAGAI, 2007), Data Mining (LIN et al., 2009), Raciocínio Baseado em Casos (CHOY; LEE, 2002), Análise Multivariada (LASCH; JANKER, 2005), Programação por metas (Goal Programming) (CHAN et al., 2010), Programação linear (DAHEL, 2003), MACBETH (OLIVEIRA; LOURENÇO, 2002), um modelo puramente matemático que utiliza abordagem multiatributo (BARLA, 2003) e os métodos desenvolvidos a partir de algoritmos bioinspirados RNA (LUO, 2009) e Ant Colony System (TSAI; YANG; LIN, 2010).

Além dos métodos simples apresentados, foram identificados modelos desenvolvidos a partir da combinação de dois ou mais métodos. O Quadro 2 lista os estudos que contemplam tais abordagens e os respectivos métodos utilizados.

A quantidade de estudos selecionados que utilizam métodos de forma isolada é de 42 (35\%), enquanto abordagens com modelos combinados totalizaram 78 estudos (65\%). Entre os estudos analisados, foram identificadas 61 diferentes combinações entre métodos MCDM, o que evidencia uma ampla gama de possibilidades a serem exploradas por novos estudos que visem otimização por meio da combinação de técnicas.

Por fim, a Figura 6 apresenta uma listagem de todos os métodos identificados e as ocorrências de uso entre os 120 estudos analisados, sejam na forma simples ou combinada.

Por meio dos resultados exibidos na Figura 6, conclui-se que os métodos que utilizam a abordagem fuzzy são os mais utilizados, já que 53 modelos
(44,16\%) incorporaram a abordagem fuzzy. Em seguida, destacam-se os métodos AHP com 32 ocorrências (26,7\%), ANP com 18 ocorrências $(15,0 \%)$, e os métodos DEA e Programação Multiobjetivo com 13 ocorrências cada um $(10,83 \%)$.

No AHP, a representação de um problema de decisão é feita em uma estrutura hierárquica de 3 níveis ("objetivo", "critérios" e "alternativas") com a finalidade de capturar os elementos básicos do problema e então derivar "índices de escala" para integrar as percepções e objetivos dentro de uma síntese. Na estrutura hierárquica, os elementos são comparados par a par com outros elementos e as comparações são utilizadas para deduzir julgamentos. A síntese dos julgamentos é obtida como um resultado da recomposição hierárquica com a finalidade de encontrar a melhor decisão (SAATY, 1980). A técnica ANP funciona de forma análoga, mas tem a vantagem de considerar dependências entre os critérios e atribuir diferentes pesos locais para os critérios em cada alternativa (KAHRAMAN, 2008).

Já a abordagem fuzzy surgiu da ausência de teorias que utilizem tratamento matemático tradicional para certas variáveis utilizadas cotidianamente, transmitidas e compreendidas linguisticamente entre interlocutores. Tais variáveis são chamadas variáveis linguísticas e são capazes de distinguir qualificações por meio de graduações ("desempenho ruim", "desempenho bom", "desempenho ótimo"). Além disso, os conjuntos cujas fronteiras são definidas por meio de propriedades subjetivas ou atributos imprecisos também podem ser tratados por este método (BARROS; BASSANEZI, 2006; CELIKYLMAZ; TÜRKSEN, 2009). Desta forma, a abordagem fuzzy é capaz de modelar variáveis do problema de seleção de fornecedores em ambientes caracterizados por ausência de informação, já que as 
Quadro 2. Listagem de métodos combinados.

\begin{tabular}{|c|c|c|}
\hline Métodos combinados & Proposto por & Quanti-dade \\
\hline Fuzzy/AHP & $\begin{array}{l}\text { Kahraman, Cebeci e Ulukan (2003), } \\
\text { Chan e Kumar (2007), } \\
\text { Yang et al. (2008), } \\
\text { Lee (2009), } \\
\text { Chamodrakas, Batis e Martakos (2010), } \\
\text { Amid, Ghodsypour e Brien (2011), } \\
\text { Kilincci e Onal (2011), } \\
\text { Punniyamoorthy, Mathiyalagan e Parthiban (2011) }\end{array}$ & 8 \\
\hline Fuzzy/ANP & $\begin{array}{l}\text { Lin (2009), } \\
\text { Önut, Kara e Isik (2009), } \\
\text { Büyüközkan e Çifçi (2011), } \\
\text { Vinodh, Ramiya e Gautham (2011) }\end{array}$ & 4 \\
\hline Programação Linear Fuzzy & $\begin{array}{l}\text { Guneri, Yucel e Ayyildiz (2009), } \\
\text { Yücel e Güneri (2011), } \\
\text { Haleh e Hamidi (2011) }\end{array}$ & 3 \\
\hline Fuzzy/QFD & $\begin{array}{l}\text { Bevilacqua, Ciarapica e Giacchetta (2006), } \\
\text { Amin e Razmi (2009) }\end{array}$ & 2 \\
\hline Fuzzy/RNA & $\begin{array}{l}\text { Kuo, Hong e Huang (2010), } \\
\text { Aksoy e Öztürk (2011) }\end{array}$ & 2 \\
\hline Fuzzy/TOPSIS & $\begin{array}{l}\text { Wang, Cheng e Kun-Cheng (2009), } \\
\text { Boran et al. (2009) }\end{array}$ & 2 \\
\hline Fuzzy/VIKOR & $\begin{array}{l}\text { Sanayei, Mousavi e Yazdankhah (2010), } \\
\text { Shemshadi et al. (2011). }\end{array}$ & 2 \\
\hline Fuzzy AHP/Programação por Metas & $\begin{array}{l}\text { Çebi e Bayraktar (2003), } \\
\text { Perçin (2006) }\end{array}$ & 2 \\
\hline Fuzzy/Programação Linear Multiobjetivo & Amid, Ghodsypour e Brien (2006) & 1 \\
\hline Fuzzy/Added-value perspective & Florez-Lopez (2007) & 1 \\
\hline Fuzzy/Cluster analysis & Bottani e Rizzi (2008) & 1 \\
\hline $\begin{array}{l}\text { Fuzzy/Specific Measurable Achievable Realistic } \\
\text { and Time-Bound (SMART) }\end{array}$ & Chou e Chang (2008) & 1 \\
\hline Fuzzy/TOPSIS/Programação Estocástica & Kara (2011) & 1 \\
\hline Fuzzy/AHP/Programação por metas/TOPSIS & Jolai et al. (2011) & 1 \\
\hline Fuzzy/Adaptive Resonance Theory & Keskin, Ilhan e Ozkan (2010) & 1 \\
\hline Fuzzy/SWOT/Programação linear & Amin, Razmi e Zhang (2011) & 1 \\
\hline Fuzzy/DEMATEL & Chang, Chang e Wu (2011) & 1 \\
\hline Fuzzy/Multi-objective goal programming/TOPSIS & Liao e Kao (2011) & 1 \\
\hline Fuzzy/Programação Multiobjetivo & Ozkok e Tiryaki (2011) & 1 \\
\hline Fuzzy/Custeio ABC & Dogan e Sahin (2003) & 1 \\
\hline Fuzzy/PROMETHEE & Araz, Ozfirat e Ozkarahan (2007) & 1 \\
\hline Fuzzy/AHP/Programação Linear & Sevkli et al. (2008) & 1 \\
\hline Fuzzy/TOPSIS/Programação linear & Songhori e Khakbaz (2009) & 1 \\
\hline Fuzzy/ELECTRE & Vadahni et al. (2010) & 1 \\
\hline AHP/Programação Linear & $\operatorname{Ng}(2008)$ & 1 \\
\hline AHP/Cluster analysis/DEA/RNA & Ha e Krishnan (2008) & 1 \\
\hline AHP/QFD & Bhattacharya, Geraghty e Young (2010) & 1 \\
\hline AHP/Fuzzy/Programação Linear Multiobjetivo & Wang e Yang (2009) & 1 \\
\hline $\begin{array}{l}\text { AHP/Algoritmo Genético/Scatter Search } \\
\text { Algorithm }\end{array}$ & Ebrahim, Razmi e Haleh (2009) & 1 \\
\hline $\begin{array}{l}\text { AHP/Multi-objective Possibilistic Linear } \\
\text { Programming/Fuzzy }\end{array}$ & Özgen et al. (2008) & 1 \\
\hline AHP/Programação Inteira Não linear & Kokangul e Susuz (2009) & 1 \\
\hline AHP/Programação dinâmica & Mafakheri, Breton e Ghoniem (2011) & 1 \\
\hline AHP/Programação por Metas/Fuzzy & Lee, Kang e Chang (2009) & 1 \\
\hline
\end{tabular}


Quadro 2. Continuação...

\begin{tabular}{|l|l|c|}
\hline \multicolumn{1}{|c|}{ Métodos combinados } & \multicolumn{1}{|c|}{ Proposto por } & Quanti-dade \\
\hline $\begin{array}{l}\text { AHP/Taguchi Loss Function/Multi-choice Goal } \\
\text { Programming }\end{array}$ & Liao e Kao (2010) & 1 \\
\hline AHP/Grey Relational Analysis (GRA) & Yang e Chen (2006) & 1 \\
\hline AHP/Programação Linear Multiobjetivo & Ting e Cho (2008) & 1 \\
\hline $\begin{array}{l}\text { ANP/Multi-objective Mixed Integer Linear } \\
\text { Programming }\end{array}$ & Ustun e Dermitas (2008a) & 1 \\
\hline ANP/Achimedean Goal Programming & Ustun e Dermitas (2008b) & 1 \\
\hline ANP/Programação Inteira Mista & Wu et al. (2009) & 1 \\
\hline ANP/Fuzzy/Programação Não linear & Razmi, Rafiei e Hashemi (2009) & 1 \\
\hline ANP/Programação Linear & Lin, Chen e Ting (2011) & 1 \\
\hline ANP/Programação Linear Inteira & Demirtas e Ustun (2008) & 1 \\
\hline ANP/TOPSIS/Nominal Group Technique (NGT) & Shyur e Shih (2006) & 1 \\
\hline ANP/Archimedean Goal Programming (AGP) & Dermitas e Ustun (2009) & 1 \\
\hline ANP/Programação Multiobjetivo & Kirytopoulos et al. (2010) & 1 \\
\hline ANP/Mixed-integer Non linear Programming & Razmi e Rafiei (2010) & 1 \\
\hline DEA/RNA & Çelebi e Bayraktar (2008) & 1 \\
\hline $\begin{array}{l}\text { DEA/RNA/Multi-attribute Decision Analysis/ } \\
\text { Linear Programming }\end{array}$ & Kuo, Wang e Tien (2010) & 1 \\
\hline DEA/SWOT/Fuzzy/TOPSIS & Chen (2011) & 1 \\
\hline DEA/Fuzzy/AHP/TOPSIS & Zeydan, Çolpan e Çobanoglu (2011) & 1 \\
\hline DEA/TCO/AHP & Ramanathan (2007) & 1 \\
\hline DEA/TCO & Garfamy (2006) & 1 \\
\hline $\begin{array}{l}\text { DEA/Árvore de decisão (Decision Trees - DT) e } \\
\text { Redes Neurais Artificiais }\end{array}$ & Wu (2009) & 1 \\
\hline DEA/Multi-objective mixed integer programming & Songhori et al. (2011) & 1 \\
\hline DEA/Programação Não linear & Hadi-Vencheh (2010) & 1 \\
\hline Multi-attribute Utility Theory/Linear Programming & Sanayei et al. (2008) & 1 \\
\hline Linear Programming/Algoritmo POT & Mendonza e Ventura (2010) & 1 \\
\hline $\begin{array}{l}\text { Programação Estocástica/Chance-Constrained } \\
\text { Programming }\end{array}$ & Li e Zabinsky (2009) & 1 \\
\hline Nonlinear Multiobjective Programming & Aguezzol e Ladet (2007) & 1 \\
\hline Raciocínio baseado em casos/RNA & Zhao e Yu (2011) & 1 \\
\hline Mixed-integer non linear Programming & Kheljani, Ghodsypour e O’brien (2009) & 1 \\
\hline & & 1 \\
\hline
\end{tabular}

pontuações dos fornecedores e os pesos dos critérios não são definidos por uma faixa de valores precisos.

Portanto, nota-se que métodos que são teoricamente mais adequados para situações de incerteza predominantemente têm recebido maior atenção por parte dos pesquisadores, posto que as abordagens fuzzy, o AHP e o ANP são os mais adotados. Comparando esses resultados com a revisão sistemática realizada por Ho, Xu e Dey (2010), conforme ilustra o Quadro 3, o amplo uso desses métodos parece ser uma tendência emergente, já que métodos matemáticos determinísticos como DEA, Programação Linear e Programação por metas anteriormente estavam entre os mais adotados. A abordagem fuzzy vem sendo amplamente adotada, pois seu uso saltou de $15,38 \%$ na revisão de Ho, Xu e Dey (2010) para 44,16\% nesta pesquisa.
Por meio do Quadro 3, também é possível constatar que o uso de métodos combinados subiu de $41,03 \%$ para $65 \%$. Como a combinação de métodos costuma ser feita com a finalidade de usufruir de algumas vantagens específicas de cada um, o aumento do uso de métodos combinados parece estar relacionado à necessidade de criar modelos capazes de tratar as peculiaridades de diferentes cenários de seleção de fornecedores.

De encontro aos resultados relativos aos métodos MCDM, está a crescente utilização de critérios qualitativos que expressam características desejáveis ao fornecedor em prol do estabelecimento de relações de "ganha-ganha" por meio da colaboração de longo prazo. O Quadro 3 apresenta alguns critérios utilizados por alguns modelos de decisão recentemente publicados pelos periódicos que registraram o 


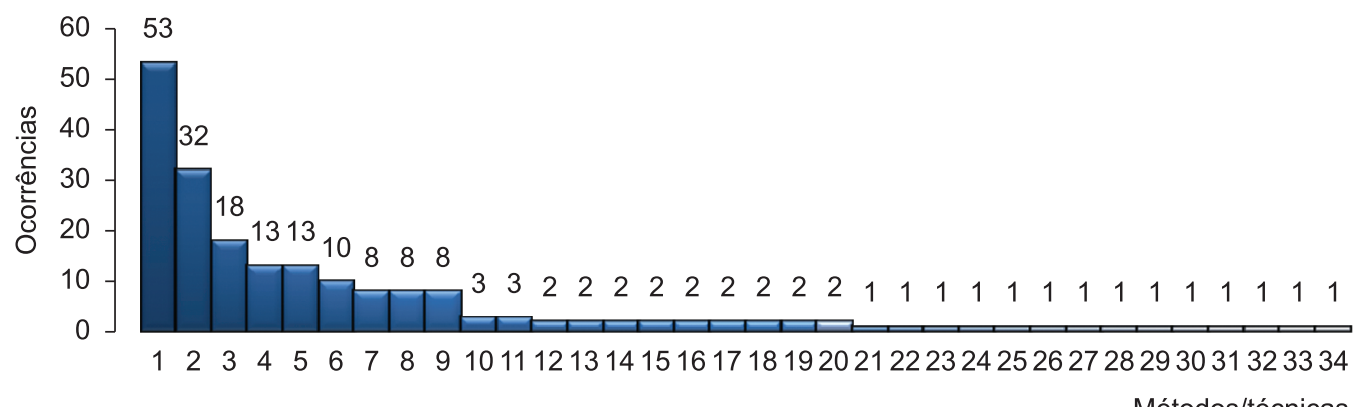

1. Abordagem Fuzzy
4. Programação multiobjetivo
7. Programação por metas
10. QFD
13. Programação dinâmica
16. TCO
19. Raciocínio baseado em casos
21. ART
24. NGT
27. Algoritmo genético
30. Data Mining
34. Support Vector Machine

2. AHP
5. DEA
8. Redes neurais artificiais
11. Programação não linear
14. Programação inteira não linear mista
17. VIKOR
20. Grey Relational Analysis
22. DEMATEL
25. PROMETHEE
28. Análise multivariada
32. Power factors aggregation method

2. AHP

8. Redes neurais artificiais

1. Programação não linear

17. VIKOR

20. Grey Relational Analysis

25. PROMETHEE

32. Power factors aggregation method
Métodos/técnicas
3. ANP
6. Programação linear
9. TOPSIS
12. Programação inteira mista
15. SWOT
18. Taguchi loss function
31. Algoritmo POT
23. ELECTRE
26. SMART
29. Ant colony system
33. Rough Set Theory

Figura 6. Quantidade de ocorrências dos métodos/técnicas identificados entre todos os estudos.

Quadro 3. Comparação dos resultados entre as revisões bibliográficas.

\begin{tabular}{|l|c|c|}
\hline \multicolumn{1}{|c|}{ Parâmetros de comparação } & Revisão de Ho, Xu e Dey (2010) & Resultados desta pesquisa \\
\hline Métodos simples mais usados & DEA, AHP e Programação linear & Fuzzy, AHP e ANP \\
\hline Método combinado mais usado & AHP/Programação por metas & Fuzzy AHP \\
\hline Uso de métodos simples & $58,97 \%$ & $35 \%$ \\
\hline Uso de métodos de combinados & $41,03 \%$ & $65 \%$ \\
\hline Quantidade de artigos analisados & 78 & 120 \\
\hline Abrangência temporal da busca & 2000 a 2008 & 2002 a 2011 \\
\hline
\end{tabular}

maior número de ocorrências (Expert Systems with Applications e International Journal Production Economics) e que foram definidos a partir de dados reais de empresas.

Conforme mostra o Quadro 4, há grande incidência de critérios relacionados ao potencial do fornecedor em inovar e desenvolver produtos conjuntamente (por exemplo, "nível de tecnologia", "poder financeiro", "construção de relacionamento", "crescimento conjunto", entre outros), bem como outros critérios qualitativos relacionados com a garantia da qualidade ("abordagem gerencial", "políticas ambientais", "habilidade de identificar necessidades", "capacidade de solucionar problemas de qualidade", entre outros). Os critérios citados, bem como diversos outros ("profissionalismo", "políticas ambientais e de segurança"), somente podem ser utilizados efetivamente em um modelo se forem adotados métodos capazes de tratar incertezas presentes nos valores numéricos que são atribuídos para essas variáveis de natureza qualitativa.
Há métodos como o fuzzy AHP, fuzzy TOPSIS e fuzzy QFD que possibilitam captar julgamentos linguísticos (por exemplo, "ruim", "bom" e "muito bom") para medir variáveis qualitativas. Um tratamento similar das variáveis é feito por sistemas de inferência fuzzy, os quais convertem valores numéricos em duas (ou mais) funções de pertinência que também representam termos linguísticos (ALTROCK, 1995; CELIKYLMAZ; TÜRKSEN, 2009). Portanto, a ampla utilização da abordagem fuzzy parece ser uma tendência que está diretamente associada à crescente adoção de critérios qualitativos emergentes, os quais expressam necessidades empresariais que visam à obtenção de vantagens por meio de uma gestão efetiva na cadeia de suprimentos e de adequações exigidas pelo cenário econômico atual (inovação, colaboração, gestão da qualidade, sustentabilidade ambiental, entre outros).

Quanto ao tipo de aplicação realizada, 70 estudos (58\%) utilizam dados fictícios para validar ou exemplificar a utilização dos modelos, enquanto $50(42 \%)$ utilizaram dados reais oriundos de 
empresas e/ou aplicaram o modelo para apoiar a seleção de fornecedores em um caso real. Quanto aos estudos que realizaram aplicação prática em empresas, a Figura 7 apresenta a listagem de setores de atuação de tais empresas pela quantidade de ocorrências.

De acordo com a Figura 7, as aplicações práticas dos métodos desenvolvidos estão fortemente direcionadas para o setor de eletroeletrônicos e tecnologia da informação. Em seguida, destaca-se o setor automotivo, o qual é historicamente pioneiro no desenvolvimento de boas práticas para a gestão de fornecedores. Com uma frequência bem menor, foram desenvolvidos estudos em empresas do setor têxtil, químico, de construção civil, alimentício e bebidas, petrolífero, de serviços, de telecomunicações, de transporte e metal-mecânico, papel e celulose. Acredita-se que características peculiares às empresas do setor de eletroeletrônicos e tecnologia da informação (afinidade com ferramentas computacionais, vasta gama de pequenos itens de matéria-prima e de fornecedores de componentes), além da ascensão deste setor na economia mundial, justifiquem a ampla adoção dos métodos MCDM.

Os resultados mostram a existência de poucos modelos desenvolvidos a partir de situações reais, salvo que a maioria dos estudos utiliza como forma de validação dos modelos a simulação com exemplos puramente numéricos. Quando são desenvolvidos modelos a partir de problemas reais identificados em empresas, os pesquisadores realizam a coleta de dados diretamente com seus representantes (encarregados de compras, desenvolvimento de produtos, gerentes de produção, entre outros atores envolvidos), definindo

Quadro 4. Alguns critérios de decisão para seleção de fornecedores.

\begin{tabular}{|l|l|}
\hline \multicolumn{1}{|c|}{ Modelo proposto por } & \multicolumn{1}{c|}{ Critérios utilizados } \\
\hline Araz e Ozkarahan (2007) & $\begin{array}{l}\text { Suporte em Desenvolvimento Estrutural de Produto, Suporte no Desenvolvimento } \\
\text { do Processo e Engenharia, Tempo de Revisão do Projeto, Tempo de Prototipagem, } \\
\text { Nível de Tecnologia, Desempenho da Qualidade, Poder Financeiro, Desempenho em } \\
\text { Redução de Custos, Desempenho de Entrega, Facilidade de Comunicação. }\end{array}$ \\
\hline Lee (2009) & $\begin{array}{l}\text { Entrega, Qualidade, Flexibilidade, Nível de Tecnologia, Construção de Relacionamento, } \\
\text { Crescimento Conjunto, Custo de Produtos, Custo de relacionamento, Restrições de } \\
\text { Suprimento, Perfil do Fornecedor e Restrições entre comprador e fornecedor. }\end{array}$ \\
\hline Shen e Yu (2009) & $\begin{array}{l}\text { Confiabilidade, Suporte técnico, Capacidade Tecnológica, Inovação, Quadro de } \\
\text { clientes, Habilidade de identificar necessidades, Velocidade de desenvolvimento dos } \\
\text { produtos. }\end{array}$ \\
\hline $\begin{array}{l}\text { Chamodrakas, Batis e } \\
\text { Martakos (2010) }\end{array}$ & $\begin{array}{l}\text { Capacidade de solucionar problemas de qualidade, Índice de Rejeição de Matéria- } \\
\text { prima, Redução de custo, Posição de Mercado do fornecedor, Compromisso com } \\
\text { prazo de entrega e Condescendência com quantidades do pedido. }\end{array}$ \\
\hline Kilincci e Onal (2011) & $\begin{array}{l}\text { Status Financeiro, Abordagem gerencial, Habilidade Técnica, Sistemas de } \\
\text { Qualidade, Localização geográfica, Capacidade de Produção, Uso de Kanban, } \\
\text { Preço do Produto, Manuseio do produto durante operações logísticas, Qualidade do } \\
\text { Produto, Lead Time, Suporte Técnico, Profissionalismo e Agilidade do Serviço. }\end{array}$ \\
\hline $\begin{array}{l}\text { Punniyamoorthy, Mathiyalagan } \\
\text { e Parthiban (2011) }\end{array}$ & $\begin{array}{l}\text { Nível de Gestão Interna, Qualidade, Capacidade Técnica, Capacidade de Produção, } \\
\text { Posição Financeira, Entrega, Serviços, Relacionamento, Custos, Políticas } \\
\text { Ambientais e de Segurança. }\end{array}$ \\
\hline
\end{tabular}

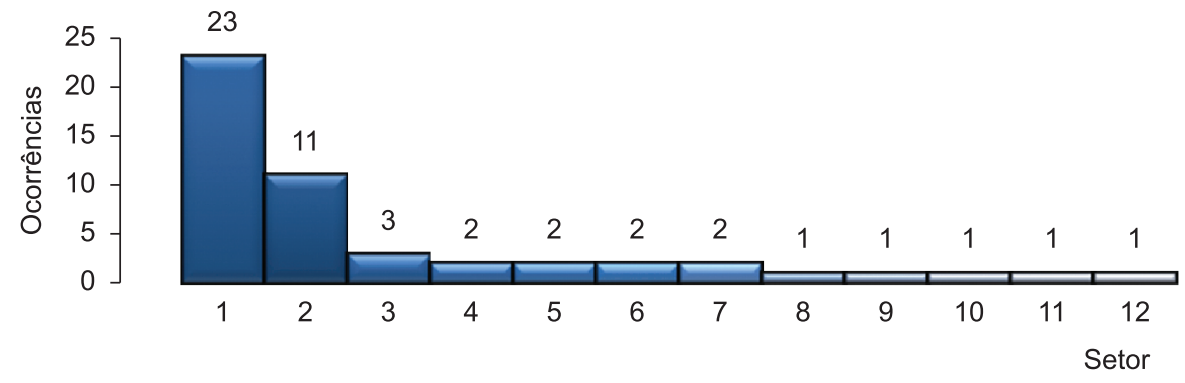
1. Eletro-eletrônicos e TI
2. Automotivo
3. Têxtil
4. Químico
5. Construção Civil
6. Alimentício e bebidas
7. Petrolífero
8. Setor de serviços
9. Telecomunicações
10. Transportes
11. Metal-mecânico
12. Papel e celulose

Figura 7. Listagem dos setores de atuação das empresas por quantidade de ocorrências. 
um conjunto de critérios a ser utilizado na avaliação das alternativas de fornecimento, o nível importância de cada um destes critérios e o desempenho das alternativas. Desta forma, tal conjunto de dados pode gerar uma modelagem bastante complexa dependendo da quantidade de critérios listados, da heterogeneidade destes e da consideração de fatores externos (geográficos e econômicos) e de questões estratégicas da rede de suprimentos. Como a maioria dos estudos analisados considerou poucos critérios e ainda de nível operacional, verifica-se que grande parte dos estudos desenvolve modelos de decisão que não são adaptáveis às reais necessidades das empresas.

\section{Considerações finais e oportunidades de pesquisa}

Por meio desta revisão sistematizada da literatura, foram identificadas algumas oportunidades para realização de pesquisas futuras, listadas no Quadro 5.

Durante a análise dos 120 artigos selecionados, foram identificadas 61 diferentes combinações de métodos MCDM para o desenvolvimento de modelos de decisão para seleção de fornecedores. Visto que foram identificados 34 técnicas de diferentes naturezas, ainda há muitas possibilidades de combinação para desenvolvimento de modelos voltados a diferentes situações de seleção e avaliação contínua de fornecedores em empresas de diferentes setores da economia que podem ser exploradas por novas pesquisas, dada a baixa ocorrência de trabalhos na maioria dos setores identificados. Conforme discutido anteriormente, para a modelagem de problemas reais, é necessário alinhar os objetivos do negócio e os requisitos dos stakeholders da empresa durante o levantamento de requisitos, considerando assim fatores estratégicos na determinação do quadro de fornecedores. Uma técnica sugerida nessas situações é o QFD (Quality Function Deployment), já que esta permite que as empresas compradoras listem requisitos desejáveis em todos os níveis da organização e estes são posteriormente desdobrados em atributos e critérios de decisão. Acredita-se que trabalhos que adotem essa técnica durante o desenvolvimento dos modelos exprimam melhor as necessidades reais do comprador.
Os resultados desta pesquisa evidenciaram que os métodos MCDM mais adequados para lidar com imprecisão e subjetividade vêm sendo os mais utilizados, estando os recursos das abordagens fuzzy presentes na maioria dos modelos. Todavia, verifica-se a necessidade de desenvolver estudos que apontem as principais semelhanças e diferenças entre esses métodos, tendo como objetivo orientar pesquisadores e profissionais durante a escolha do(s) método(s) e o desenvolvimento de modelos sob o contexto de seleção de fornecedores.

Salminen, Hokkanen e Lahdelma (1998) compararam os métodos ELECTRE, PROMETHEE e SMART; Mahmoud e Garcia (2000) compararam PROMETHEE, ELECTRE e AHP; Bhutta e Huq (2002) compararam os métodos AHP e TCO; Ertugrul e Karakasoglu (2007) compararam fuzzy TOPSIS e fuzzy AHP, sendo todos os estudos realizados sob o contexto de problemas de decisão diferentes do processo de seleção de fornecedores. Todos esses estudos foram conduzidos a partir da realização de uma pesquisa bibliográfica detalhada seguida pelo desenvolvimento de modelos, por simulações de uso e, posteriormente, pela análise e comparação dos modelos segundo parâmetros previamente definidos. Porém, é válido ressaltar que as peculiaridades dos problemas em que o método MCDM é aplicado influencia diretamente seu desempenho durante a comparação. Somente o estudo de Bhutta e Huq (2002) realizou um estudo comparativo sob a ótica do problema de seleção de fornecedores. Não foram encontrados estudos que comparem a abordagem fuzzy, AHP e ANP sob o contexto do problema de seleção de fornecedores, evidenciando assim uma lacuna na literatura científica relacionada.

Quanto à definição e ao uso de alguns termos básicos referentes ao presente tema de pesquisa, a partir da revisão bibliográfica realizada, o autor optou por referenciar cada uma das abordagens propostas pelos estudos analisados como "modelos MCDM", considerando que estes são baseados em métodos MCDM e outras técnicas de diversas naturezas, por esta forma de conceituação ser mais coerente com a literatura de pesquisa operacional e simulação. No entanto, alguns estudos referenciam esses modelos como "métodos MCDM", "técnicas multicritério", "abordagens multicritério", "análise multicritério" ou ainda "ferramentas multicritério".

Quadro 5. Oportunidades para realização de pesquisas futuras.

Explorar novas combinações entre métodos MCDM e técnicas já existentes.

Desenvolver modelos MCDM para empresas de setores pouco explorados, como telecomunicações, transportes, papel e celulose, metal-mecânico e serviços.

Comparar métodos MCDM sob o contexto de seleção de fornecedores.

Desenvolver ontologias voltadas para tomada de decisão multicritério. 
Também foi observado que não há consenso entre os estudos ao usar os conceitos "critérios de decisão", "subcritérios", "atributos", "clusters" e "fatores de medição", principalmente nos estudos que utilizam AHP. Em alguns casos, o conjunto de nós que envolve um elemento do segundo nível da estrutura e os demais elementos do terceiro nível ligados a este é considerado como um "critério" que se desdobra em "atributos", "subcritérios" ou "fatores de medição". Já em outros casos, tal conjunto de elementos é chamado de cluster ou "fatores de medição", sendo os elementos de terceiro nível referenciados como "critérios" ou "atributos". É evidente que a forma de denominação dos elementos em uma estrutura de hierarquia não irá influenciar o resultado do processo decisório. Contudo, como qualquer tema de pesquisa, é importante que os termos sejam utilizados na literatura de forma padronizada em prol da solidificação do tema de pesquisa e melhor entendimento dos pesquisadores e profissionais da área. Desta forma, o desenvolvimento de ontologias voltadas para a modelagem de problemas de decisão se apresenta como uma oportunidade de pesquisa.

Os resultados mostraram que a maioria dos artigos sobre o tema de pesquisa analisado é originária de Taiwan, da Turquia, do Irã e da China e que estudos de origem brasileira sobre este tema não foram localizados durante as buscas detalhadas na Seção 3. Depois desta constatação, outras buscas foram realizadas com a finalidade de investigar a existência de estudos de origem brasileira. Nenhum estudo foi encontrado durante as consultas realizadas no portal de periódicos Scielo (www.scielo.org). Contudo, a partir de buscas realizadas em outras bases de dados, foram localizadas algumas dissertações de mestrado e trabalhos publicados em anais de eventos.

Ribeiro (2008) desenvolveu um modelo que apoia a etapa de escolha final de fornecedores e também a avaliação contínua dos selecionados utilizando os métodos ANP e AHP; Salomon, Marins e Duduch (2007) propuseram um modelo AHP voltado para uma indústria automotiva; Alencar e Almeida (2010) utilizam ELECTRE IV e VIP Analysis para apoiar a seleção de fornecedores em projetos em grupo; Diante desses resultados, nota-se que no Brasil vêm sendo desenvolvidos estudos neste tema e que estes adotam métodos MCDM da escola francesa (ELECTRE I e IV) e da escola americana (AHP, ANP e MACBETH). Porém, não foram identificados estudos que utilizem técnicas de IA.

O relato de casos de aplicação de modelos de decisão para seleção de fornecedores, bem como para sua avaliação contínua, ainda é pouco recorrente na literatura. No Brasil, a realização de tais pesquisas costuma ser dificultada pela falta de confiança de algumas empresas que se negam a fornecer informações relacionadas ao problema, pela pouca disponibilidade dos agentes decisórios para validar os modelos propostos e pelo distanciamento entre as decisões racionais e as práticas reais adotadas para seleção de fornecedores. Contudo, cabe aos pesquisadores o esforço de difundir esses conhecimentos para que os métodos MCDM possam ser incorporados a softwares de compra, de gestão integrada, ou mesmo implementados em planilhas simples e de fácil manipulação, contribuindo para que as empresas determinem racionalmente a composição da sua base de fornecedores e, dadas a grande importância e complexidade da decisão, se tornem mais competitivas por meio disso.

Em síntese, o número de pesquisas sobre o tema vem crescendo, mas, no Brasil, ainda existem poucos estudos e estes pouco exploram a diversidade de métodos existentes. Os métodos MCDM mais utilizados são aqueles capazes de lidar com incertezas, o que condiz com a tendência de adotar critérios qualitativos que exprimem adequações necessárias às empresas diante do atual cenário econômico marcado por uma série de transformações nos paradigmas de produção. Apesar de a amostra possuir um tamanho de 120 artigos, acredita-se que esta seja capaz de representar o estado da arte do tema, dadas a rigidez dos procedimentos utilizados nas buscas, a utilização de 4 portais de periódicos de bastante relevância e a seleção de estudos publicados nos últimos 10 anos.

\section{Agradecimentos}

À Fundação de Amparo à Pesquisa do Estado de São Paulo e à Coordenação de Aperfeiçoamento de Pessoal de Nível Superior pelo financiamento desta pesquisa.

\section{Referências}

AGUEZZOL, A.; LADET, P. A nonlinear multiobjective approach for the supplier selection, integrating transportation policies. Journal of Modelling in Management, v. 2, p. 157-169, 2007. http://dx.doi. org/10.1108/17465660710763434

AISSAOUI, N.; HAOUARI, M.; HASSINI, E. Supplier selection and order lot sizing modeling: A review. Computers \& Operations Research, v. 34, p. 35163540, 2007. http://dx.doi.org/10.1016/j.cor.2006.01.016 AKSOY, A.; ÖZTÜRK, N. Supplier selection and performance evaluation in just-in-time production environments. Expert Systems with Applications, v. 38, p. 6351-359, 2011. http://dx.doi.org/10.1016/j. eswa.2010.11.104

ALBINO, V.; GARAVELLI, A. C. A neural network application to subcontractor rating in construction. International Journal of Project Management, v. 16, p. 9-14, 1998. http://dx.doi.org/10.1016/ S0263-7863(97)00007-0

ALENCAR, L. H.; ALMEIDA, A. T. Suppliers Selection based on Multicriteria Group Decision Model. In: 
GROUP DECISION AND NEGOTIATION - GDN, 2010, Delft. Procedings... GDN, 2010.

ALTROCK, C. V. Fuzzy Logic and Neurofuzzy - Applications in Business and Finance. Prentice Hall: USA, 1995.

AMBROSE, E. et al. Communication media selection in buyer-supplier relationships. International Journal of Operations \& Production Management, v. 28, p. 360-379, 2008. http://dx.doi. org/10.1108/01443570810861561

AMID, A. A.; GHODSYPOUR, S. H.; BRIEN, C. O. Fuzzy multiobjective linear model for supplier selection in supply chain. International Journal of Production Economics, v. 104, p. 394-407, 2006. http://dx.doi. org/10.1016/j.ijpe.2005.04.012

AMID, A. A.; GHODSYPOUR, S. H.; BRIEN, C. O. A weighted max-min model for fuzzy multiobjective supplier selection in a supply chain. International Journal of Production Economics, v. 131, p. 139-145, 2011. http://dx.doi.org/10.1016/j. ijpe.2010.04.044

AMIN, S. H.; RAZMI, J. An integrated fuzzy model for supplier management: A case study of ISP selection and evaluation. Expert Systems with Applications, v. 36, p. 8639-8648, 2009. http://dx.doi.org/10.1016/j. eswa.2008.10.012

AMIN, S. H.; RAZMI, J.; ZHANG, G. Supplier selection and order allocation based on fuzzy SWOT analysis and fuzzy linear programming. Expert Systems with Applications, v. 38, p. 334-342, 2011. http://dx.doi. org/10.1016/j.eswa.2010.06.071

ANDRADE, E. L. Introdução à Pesquisa Operacional - Métodos e modelos para tomada de decisão. Rio de Janeiro: LTC, 1998.

ARAZ, C.; OZFIRAT, P. M.; OZKARAHAN, I. An integrated multicriteria decision-making methodology for outsourcing management. Computers \& Operations Research, v. 34, p. 3738-3756, 2007. http://dx.doi. org/10.1016/j.cor.2006.01.014

ARAZ, C.; OZKARAHAN, I. Supplier evaluation and management system for strategic sourcing based on a new multicriteria sorting procedure. International Journal of Production Economics, v. 106, p. 585-606, 2007. http://dx.doi.org/10.1016/j.ijpe.2006.08.008

BARLA, S. B. A case study of supplier selection for lean supply by using a mathematical model. Logistics Information Management, v. 16, p. 451-459, 2003. http://dx.doi.org/10.1108/09576050310503420

BARROS, L. C.; BASSANEZI, E. R. Tópicos de Lógica Fuzzy e Biomatemática. Campinas: Editora do IMECC-UNICAMP, 2006.

BENAYOUN, R.; ROY, B.; SUSSMAN, B. ELECTRE: Une méthod e pour guider le choix en présence de points de vue multiples. Paris: SEMAMETRA International, Direction Scientifique, 1966. Note de travail, n. 49.

BEVILACQUA, M.; CIARAPICA, F. E.; GIACCHETTA, G. A fuzzy-QFD approach to supplier selection. Journal of Purchasing \& Supply Management, v. 12, p. 14-27, 2006. http://dx.doi.org/10.1016/j. pursup.2006.02.001

BHATTACHARYA, A.; GERAGHTY, J.; YOUNG, P. Supplier selection paradigm: An integrated hierarchical QFD methodology under multiple-criteria environment.
Applied Soft Computing, v. 10, p. 1013-1027, 2010. http://dx.doi.org/10.1016/j.asoc.2010.05.025

BHUTTA, K. S.; HUQ, F. Supplier selection problem: a comparison of the Total Cost Ownership and Analytic Hierarchy Process approaches. Supply Chain Management, v. 7, p. 126-135, 2002. http://dx.doi. org/10.1108/13598540210436586

BORAN, F. E. et al. A multi-criteria intuitionistic fuzzy group decision making for supplier selection with TOPSIS method. Expert Systems with Applications, v. 36, p. 11363-11368, 2009. http://dx.doi.org/10.1016/j. eswa.2009.03.039

BOTTANI, E.; RIZZI, A. An adapted multi-criteria approach to suppliers and products selection - An application oriented to lead-time reduction. International Journal of Production Economics, v. 111, p. 763-781, 2008. http://dx.doi.org/10.1016/j.ijpe.2007.03.012

BÜYÜKÖZKAN, G.; ÇIFÇI, G. A novel fuzzy multi-criteria decision framework for sustainable supplier selection with incomplete information. Computers in Industry, v. 62, p. 164-174, 2011. http://dx.doi.org/10.1016/j. compind.2010.10.009

CASTRO, W. A. S.; GOMEZ, O. D. C.; FRANCO, L. F. O. Seleccion de proveedores: uma aproximacion al estado del arte. Cuadernos de Administración, v. 22, p. 145-167, 2009.

ÇEBI, F.; BAYRAKTAR, D. An integrated approach for supplier selection. Logistics Information Management, v. 16, p. 395-400, 2003. http://dx.doi. org/10.1108/09576050310503376

ÇELEBI, D.; BAYRAKTAR, D. An integrated neural network and data envelopment analysis for supplier evaluation under incomplete information. Expert Systems with Applications, v. 35, p. 1698-1710, 2008. http://dx.doi.org/10.1016/j.eswa.2007.08.107

CELIKYLMAZ, A.; TÜRKSEN, I. B. Modeling Uncertainty with Fuzzy Logic with recent theory and applications. Springer, 2009. http://dx.doi. org/10.1007/978-3-540-89924-2

CHAMODRAKAS, I.; BATIS, D.; MARTAKOS, D. Supplier selection in electronic marketplaces using satisficing and fuzzy AHP. Expert Systems with Applications, v. 37, p. 490-498, 2010. http://dx.doi.org/10.1016/j. eswa.2009.05.043

CHAN, F. T. S.; KUMAR, N. Global supplier development considering risk factors using fuzzy extended AHP-based approach. Omega, v. 35, p. 417-431, 2007. http://dx.doi. org/10.1016/j.omega.2005.08.004

CHAN, F. T. S. et al. B2B multi-attribute e-procurement: an artificial immune system based goal programming approach. International Journal of Production Research, v. 49, p. 321-341, 2010. http://dx.doi. org/10.1080/00207540902922802

CHANG, B.; CHANG, C.; WU, C. Fuzzy DEMATEL method for developing supplier selection criteria. Expert Systems with Applications, v. 38, p. 1850-1858, 2011. http://dx.doi.org/10.1016/j.eswa.2010.07.114

CHANG, B.; HUNG, H. A study of using RST to create the supplier selection model and decision-making rules. Expert Systems with Applications, v. 37, p. 82848295, 2010. http://dx.doi.org/10.1016/j.eswa.2010.05.056

CHARNES, A.; COOPER, W. W.; RHODES, E. Measuring the efficiency of decision making units. European 
Journal of Operational Research, v. 2, p. 429-444, 1978. http://dx.doi.org/10.1016/0377-2217(78)90138-8

CHE, Z. H.; WANG, H. S. Supplier selection and supply quantity allocation of common and non-common parts with multiple criteria under multiple products. Computers \& Industrial Engineering, v. 55, p. 110-133, 2008. http://dx.doi.org/10.1016/j.cie.2007.12.005

CHEN, C.; LIN, C.; HUANG, S. A fuzzy approach for supplier evaluation and selection in supply chain management. International Journal of Production Economics, v. 102, p. 289-301, 2006. http://dx.doi. org/10.1016/j.ijpe.2005.03.009

CHEN, W. Structured methodology for supplier selection and evaluation in a supply chain. Information Sciences, v. 181, p. 1651-1670, 2011. http://dx.doi.org/10.1016/j. ins.2010.07.026

CHOU, S. Y.; SHEN, C. Y.; CHANG, Y. H. Vendor Selection in a modified re-buy situation using a strategy-aligned fuzzy approach. International Journal of Production Research, v. 45, p. 3113-3133, 2007. http://dx.doi. org/10.1080/00207540600806489

CHOU, S.; CHANG, Y. A decision support system for supplier selection based on a strategy-aligned fuzzy SMART approach. Expert Systems with Applications, v. 34, p. 2241-2253, 2008. http://dx.doi.org/10.1016/j. eswa.2007.03.001

CHOY, K. L.; LEE, W. B. A generic tool for selection and management of supplier relationship in a outsourced manufacturing environment: the application of case based reasoning. Logistics Information Management, v. 15, p. 235-253, 2002. http://dx.doi. org/10.1108/09576050210436093

CHRISTOPHER, M. (Ed.). Logistics: The Strategic Issues. London: Chapman and Hall, 1992. p. 63-75.

DAHEL, N. Vendor selection and order quantity allocation in volume discount environments. Supply Chain Management, v. 8, p. 335-342, 2003. http://dx.doi. org/10.1108/13598540310490099

DANTZIG, G. Linear Programming and Extensions. Princeton: Princeton University Press, 1963.

DAS, D.; ROY, A.; KAR, S. Improving production policy for a deteriorating item under permissible delay in payments with stock-dependent demand rate. Computers and Mathematics with Applications, v. 60, p. 1973-1985, 2010. http://dx.doi.org/10.1016/j. camwa.2010.07.031

DE BOER, L.; PIERANGELA, E.; MORLACCHI, P. A review of methods supporting supplier selection. European Journal of Purchasing \& Supply Management, v. 7, p. 75-89, 2001. http://dx.doi. org/10.1016/S0969-7012(00)00028-9

DE BOER, L.; WEGEN, L. V. D.; TELGEN, J. Outranking methods in support of supplier selection. European Journal of Purchasing \& Supply Management, v. 4, p. 109-118, 1998. http://dx.doi.org/10.1016/ S0969-7012(97)00034-8

DEGRAEVE, Z.; LABRO, E.; ROODHOOFT, F. An evaluation of supplier selection methods from a total cost of ownership perspective. European Journal of Operational Research, v. 125, p. 34-58, 2000. http:// dx.doi.org/10.1016/S0377-2217(99)00199-X

DEMIRTAS, E. A.; USTUN, O. An integrated multiobjective decision making process for supplier selection and order allocation. Omega, v. 36, p. 76-90, 2008. http://dx.doi. org/10.1016/j.omega.2005.11.003

DERMITAS, E. A.; USTUN, O. Analytic network process and multi-period goal programming integration in purchasing decisions. Computers \& Industrial Engineering, v. 56, p. 677-690, 2009. http://dx.doi. org/10.1016/j.cie.2006.12.006

DICKSON, G. W. An analysis of vendor selection systems and decisions. Journal of Purchasing, v. 2, p. 5-17, 1966.

DOGAN, I.; SAHIN, U. Supplier selection using activitybased costing and fuzzy presentworth techniques. Logistics Information Management, v. 16, p. 420-426, 2003. http://dx.doi.org/10.1108/09576050310503394

DUMMING, R.; MINNINO, V. Supplier selection using a multi-criteria decision aid method. Journal of Purchasing \& Supply Management, v. 9, p. 177-187, 2003. http:// dx.doi.org/10.1016/S1478-4092(03)00032-3

EBRAHIM, R. M.; RAZMI, J.; HALEH, H. Scatter search algorithm for supplier selection and order lot sizing under multiple price discount environment. Advances in Engineering Software, v. 40, p. 766-776, 2009. http:// dx.doi.org/10.1016/j.advengsoft.2009.02.003

ERTUGRUL, I.; KARAKASOGLU, N. Comparision of fuzzy AHP and fuzzy TOPSIS methods for facility location selection. International Journal Advances Manufacturing Technology, v. 39, p. 783-795, 2008. http://dx.doi.org/10.1007/s00170-007-1249-8

FIGUEIRA, J.; GRECO, S.; EHRGOTT, M. Criteria Decision Analysis. Springer Science, 2005. 1085 p. PMCid:1739777.

FLOREZ-LOPEZ, R. Strategic supplier selection in the added-value perspective: A CI approach. Information Sciences, v. 177, p. 1169-1179, 2007. http://dx.doi. org/10.1016/j.ins.2006.08.009

FRÖDELL, M. Criteria for achieving efficient contractorsupplier relations. Engineering, Construction and Architectural Management, v. 18, p. 381-393, 2011. http://dx.doi.org/10.1108/09699981111145826

GARFAMY, R. M. A data envelopment analysis approach based on total cost of ownership for supplier selection. Journal of Enterprise Information, v. 6, p. 662-678, 2006. http://dx.doi. org/10.1108/17410390610708526

GENCER, C.; GÜRPINAR, D. Analytic network process in supplier selection: A case study in an electronic firm. Applied Mathematical Modelling, v. 31, p. 24752486, 2007. http://dx.doi.org/10.1016/j.apm.2006.10.002

GOMES, L. F. A. M. Pesquisa Operacional na Tomada de Decisões. São Paulo: Campus, 2004. 384 p.

GONZÁLEZ, M. E. et al. Determining the importance of supplier selection process in manufacturing: a case study. International. Journal of Physical Distribution \& logistics Management, v. 34, p. 492-504, 2004. http:// dx.doi.org/10.1108/09600030410548550

GUNERI, A. F.; YUCEL, A.; AYYILDIZ, G. An integrated fuzzy-LP approach for a supplier selection problem in supply chain management. Expert Systems with Applications, v. 36, p. 9223-9228, 2009. http://dx.doi. org/10.1016/j.eswa.2008.12.021

GUO, X.; YUAN, Z.; TIAN, B. Supplier selection based on hierarchical potential support vector machine. Expert Systems with Applications, v. 36, p. 6978-6985, 2009. http://dx.doi.org/10.1016/j.eswa.2008.08.074 
HA, S. H.; KRISHNAN, R. A hybrid approach to supplier selection for the maintenance of a competitive supply chain. Expert Systems with Applications, v. 34, p. 13031311, 2008. http://dx.doi.org/10.1016/j.eswa.2006.12.008

HADI-VENCHEH, A. A new nonlinear model for multiple criteria supplier selection problem. International Journal of Computer Integrated Manufacturing, v. 24 , p. 32-39, 2010. http://dx.doi.org/10.1080/0951 192X.2010.527372

HALEH, H.; HAMIDI, A. A fuzzy MCDM model for allocating orders to suppliers in a supply chain under uncertainty over a multi-period time horizon. Expert Systems with Applications, v. 38, p. 9076-9083, 2011. http://dx.doi.org/10.1016/j.eswa.2010.11.064

HINKLE, C. L.; ROBINSON, P. J.; GREEN, P. E. Vendor evaluation using cluster analysis. Journal of Purchasing, p. 49-58, 1969.

HO, W.; XU, X.; DEY, P. K. Multi-criteria decision making approaches for supplier evaluation and selection: A literature review. European Journal of Operational Research, v. 202, p. 16-24, 2010. http://dx.doi. org/10.1016/j.ejor.2009.05.009

HOLLAND, J. H. Genetic Algorithms. Scientific American, v. 267 , p. 44-50, 1992. http://dx.doi.org/10.1038/ scientificamerican0792-66

HOU, J.; SU, D. Integration of Web Services technology with business models within. Computers in Industry, v. 57, p. 797-808, 2006. http://dx.doi.org/10.1016/j. compind.2006.04.008

HSU, B.; CHIANG, C.; SHU, M. Supplier selection using fuzzy quality data and their applications to touch screen. Expert Systems with Applications, v. 37, p. 6192-6200, 2010. http://dx.doi.org/10.1016/j. eswa.2010.02.106

HSU, C.; HU, A. H. Applying hazardous substance management to supplier selection. Journal of Cleaner Production, v. 17, p. 255-264, 2009. http://dx.doi. org/10.1016/j.jclepro.2008.05.004

JOLAI, F. et al. Integrating fuzzy TOPSIS and multi-period goal programming for purchasing multiple products from multiple suppliers. Journal of Purchasing \& Supply Management, v. 17, p. 42-53, 2011. http:// dx.doi.org/10.1016/j.pursup.2010.06.004

KAHRAMAN, C. Fuzzy Multicriteria Decision Making - Theory and Applications with Recent Developments. Turkey: Springer Science, 2008.

KAHRAMAN, C.; CEBECI, U.; ULUKAN, Z. Multi-criteria supplier selection using fuzzy. Logistics Information Management, v. 16, p. 382-394, 2003. http://dx.doi. org/10.1108/09576050310503367

KARA, S. S. Supplier selection with an integrated methodology in unknown environment. Expert Systems with Applications, v. 38, p. 2133-2139, 2011. http:// dx.doi.org/10.1016/j.eswa.2010.07.154

KEENEY, R. L.; RAIFFA, H. Decision with Multiple Objectives: Preferences and Value Trade-offs. New York: John Wiley \& Sons, 1976.

KESKIN, G. A.; ILHAN, S.; OZKAN, C. The Fuzzy ART algorithm: A categorization method for supplier evaluation and selection. Expert Systems with Applications, v. 37, p. 1235-1240, 2010. http://dx.doi. org/10.1016/j.eswa.2009.06.004

KHELJANI, J. G.; GHODSYPOUR, G. H.; O'BRIEN, C. Optimizing whole supply chain benefit versus buyer's benefit through supplier selection. International Journal of Production Economics, v. 121, p. 482-493, 2009. http://dx.doi.org/10.1016/j.ijpe.2007.04.009

KILINCCI, O.; ONAL, S. A. Fuzzy AHP approach for supplier selection in a washing machine company. Expert Systems with Applications, v. 38, p. 9656-9664, 2011. http://dx.doi.org/10.1016/j.eswa.2011.01.159

KIRYTOPOULOS, K. et al. Multiple sourcing strategies and order allocation: an ANP-AUGMECON metamodel. Supply Chain Management: An International Journal, v. 15, p. 263-276, 2010. http://dx.doi. org/10.1108/13598541011054643

KIRYTOPOULOS, K.; LEOPOULOS, V.; VOULGARIDOU, D. Supplier selection in pharmaceutical industry. Benchmarking: An International Journal, v. 15, p. 494-516, 2008.

KITCHENHAM, B. A.; CHARTERS, S. Guidelines for performing Systematic Literature Reviews. EBSE, 2007. Software Engineering Technical Report EBSE-2007-01.

KLIR, G. J. Fuzzy Sets and Fuzzy Logic: theory and applications. London: Prentice Hall, 1995.

KOKANGUL, A.; SUSUZ, Z. Integrated analytical hierarch process and mathematical programming to supplier selection problem with quantity discount. Applied Mathematical Modelling, v. 33, p. 1417-1429, 2009. http://dx.doi.org/10.1016/j.apm.2008.01.021

KUO, R. J.; HONG, S. Y.; HUANG, Y. C. Integration of particle swarm optimization-based fuzzy neural network and artificial neural network for supplier selection. Applied Mathematical Modelling, v. 34, p. 39763990, 2010. http://dx.doi.org/10.1016/j.apm.2010.03.033

KUO, R. J.; WANG, Y. C.; TIEN, F. C. Integration of artificial neural network and MADA methods for green supplier selection. Journal of Cleaner Production, v. 18, p. 1161-1170, 2010. http://dx.doi.org/10.1016/j. jclepro.2010.03.020

LAM, K.; TAO, R.; LAM. M. C. A material supplier selection model for property developers using Fuzzy Principal Component Analysis. Automation in Construction, v. 19, p. 608-618, 2010. http://dx.doi.org/10.1016/j. autcon.2010.02.007

LASCH, R.; JANKER, C. G. Supplier selection and controlling using multivariate analysis. International Journal of Physical Distribution \& Logistics Management, v. 35, p. 409-425, 2005. http://dx.doi. org/10.1108/09600030510611648

LEE, A. H. I. A fuzzy supplier selection model with the consideration of benefits, opportunities, costs and risks. Expert Systems with Applications, v. 36, p. 2879 2893, 2009. http://dx.doi.org/10.1016/j.eswa.2008.01.045

LEE, A. H. I.; CHANG, H.; LIN C. An evaluation model of buyer-supplier relationships in high-tech industry - The case of an electronic components manufacturer in Taiwan. Computers \& Industrial Engineering, v. 57, p. 1417 1430, 2009. http://dx.doi.org/10.1016/j.cie.2009.07.012

LEE, A. H. I.; KANG, H.; CHANG, C. Fuzzy multiple goal programming applied to TFT-LCD supplier selection by downstream manufacturers. Expert Systems with Applications, v. 36, p. 6318-6325, 2009. http://dx.doi. org/10.1016/j.eswa.2008.08.044

LI, G.; YAMAGUCHI, D.; NAGAI, M. A grey-based decision-making approach to the supplier selection problem. Mathematical and Computer Modelling, 
v. 46, p. 573-581, 2007. http://dx.doi.org/10.1016/j. mcm.2006.11.021

LI, L.; ZABINSKY, Z. B. Incorporating uncertain into a supplier selection problem. International Journal of Production Economics, v. 134, n. 2, p. 344-356, 2009. http://dx.doi.org/10.1016/j.ijpe.2009.11.007

LIAO, C.; KAO, H. Supplier selection model using Taguchi loss function, analytical hierarchy process and multichoice goal programming. Computers \& Industrial Engineering, v. 58, p. 571-577, 2010. http://dx.doi. org/10.1016/j.cie.2009.12.004

LIAO, C.; KAO, H. An integrated fuzzy TOPSIS and MCGP approach to supplier selection in supply chain management. Expert Systems with Applications, v. 38, n. 9, p. 10803-10811, 2011. http://dx.doi.org/10.1016/j. eswa.2011.02.031

LIAO, Z.; RITTSCHER, J. Integration of supplier selection, procurement lot sizing and carrier selection under dynamic demand conditions. International Journal of Production Economics, v. 107, p. 502-510, 2007. http://dx.doi.org/10.1016/j.ijpe.2006.10.003

LIN, C.; CHEN, C.; TING, Y. An ERP model for supplier selection in electronics industry. Expert Systems with Applications, v. 38, p. 1760-1765, 2011. http://dx.doi. org/10.1016/j.eswa.2010.07.102

LIN, R. An integrated FANP-MOLP for supplier evaluation and order allocation. Applied Mathematical Modelling, v. 33, p. 2730-2736, 2009. http://dx.doi.org/10.1016/j. apm.2008.08.021

LIN, R. et al. An integrated method for finding key suppliers in SCM. Expert Systems with Applications, v. 36, p. 6461-6465, 2009. http://dx.doi.org/10.1016/j. eswa.2008.07.078

LUO, X. Supplier selection in agile supply chains: an information-processing model and an illustration. Journal of Purchasing \& Supply Management, v. 15, p. 249-262, 2009. http://dx.doi.org/10.1016/j. pursup.2009.05.004

MAFAKHERI, F. M.; BRETON, M.; GHONIEM, A. Supplier selection-order allocation: A two-stage multiple criteria dynamic programming approach. International Journal of Production Economics, v. 132, n. 1, p. 52-57, 2011. http://dx.doi.org/10.1016/j. ijpe.2011.03.005

MAHMOUD, M. R.; GARCIA, L. A. Comparison of different multicriteria evaluation methods for the Red Bluff diversion dam. Environment Modelling \& Software, v. 15, p. 471-478, 2000. http://dx.doi. org/10.1016/S1364-8152(00)00025-6

MENDONZA, A.; VENTURA, J. A. A serial inventory system with supplier selection and order quantity allocation. European Journal of Operational Research, v. 207, p. 1304-1315, 2010. http://dx.doi.org/10.1016/j. ejor.2010.06.034

MICHELI, G. J. L. A decision-maker-centred supplier selection approach for critical supplies. Management Decision, v. 46, p. 918-932, 2008. http://dx.doi. org/10.1108/00251740810882671

MONCZKA, R. M.; TRECHA, S. J. Cost-based supplier performance evaluation. Journal of Purchasing and Materials Management, v. 24, p. 2-7, 1988.

MOORE, D. L.; FEARON, H. E. Computer-assisted decision-making in purchasing. Journal of Purchasing, v. 9, p. 5-25, 1972.
MOORE, J. H.; WEATHERFORD, L. R. Tomada de Decisão em Administração. 6. ed. Porto Alegre: Bookman, 2005.

MORLACCHI, P. Small and medium enterprises in supply chain: a supplier evaluation model and some empirical results. In: IFPMM SUMMER SCHOOL, 1997, Saltzburg. Proceedings... Saltzburg: IFPMM, 1997.

NARASIMHAN, R. An analytic approach to supplier selection. Journal of Purchasing and Supply Management, v. 1, p. 27-32, 1983.

NDUBISI, N. O. et al. Supplier Selection and Management Strategies and Manufacturing Flexibility. The Journal of Enterprise Information Management, v. 18, p. 330-349, 2005. http://dx.doi. org/10.1108/17410390510592003

NG, W. L. An efficient and simple model for multiple criteria supplier selection problem. European Journal of Operational Research, v. 186, p. 1059-1067, 2008. http://dx.doi.org/10.1016/j.ejor.2007.01.018

OLIVEIRA, R. C.; LOURENÇO, J. C. A multicriteria model for assigning new orders to service suppliers. European Journal of Operational Research, v. 139 , p. $390-399,2002$. http://dx.doi.org/10.1016/ S0377-2217(01)00367-8

OLIVER, R. K.; WEBBER, M. D. Supply-chain management: logistics catches up with strategy. Outlook, Booz, Allen and Hamilton Inc. Reprinted 1992. In: CHRISTOPHER, M. (Ed.). Logistics: The Strategic Issues. London: Chapman Hall, 1982. p. 63-75.

ÖNUT, S.; KARA, S. S.; ISIK, E. Long term supplier selection using a combined fuzzy MCDM approach: A case study for a telecommunication company. Expert Systems with Applications, v. 36, p. 3887-3895, 2009. http://dx.doi.org/10.1016/j.eswa.2008.02.045

ORDOOBADI, S. M. Development of a supplier selection model using fuzzy logic. Supply Chain Management: An International Journal, v. 14, p. 314-327, 2008. http:// dx.doi.org/10.1108/13598540910970144

ORDOOBADI, S. M. Application of Taguchi loss functions for supplier selection. Supply Chain Management: An International Journal, v. 14, p. 22-30, 2009. http:// dx.doi.org/10.1108/13598540910927278

ÖZGEN, D. et al. A two-phase possibilistic linear programming methodology for multi-objective supplier evaluation and order allocation problems. Information Sciences, v. 178, p. 485-500, 2008. http://dx.doi. org/10.1016/j.ins.2007.08.002

OZKOK, B. A.; TIRYAKI, F. A compensatory fuzzy approach to multi-objective linear supplier selection problem with multiple-item. Expert Systems with Applications, v. 38, n. 9, p. 11363-11368, 2011. http:// dx.doi.org/10.1016/j.eswa.2011.03.004

PERÇIN, S. An application of the integrated AHP-PGP model in supplier selection. Measuring Business Excellence, v. 10, p. 34-49, 2006. http://dx.doi. org/10.1108/13683040610719263

PIDDUCK, A. B. Issues in supplier partner selection. Journal of Enterprise Information Management, v. 19, p. 262-276, 2006. http://dx.doi. org/10.1108/17410390610658450

PRESSEY, A. D.; WINKLHOFER, H.; TZOKAS, N. $\mathrm{X}$. Purchasing practices in small- to medium-sized enterprises: An examination of strategic purchasing adoption, supplier evaluation and supplier capabilities. 
Journal of Purchasing and Supply Management, v. 15 , p. 214-226, 2009. http://dx.doi.org/10.1016/j. pursup.2009.03.006

PUNNIYAMOORTHY, M.; MATHIYALAGAN, P.; PARTHIBAN, P. A strategic model using structural equation modeling and fuzzy logic in supplier selection. Expert Systems with Applications, v. 38, p. 458-474, 2011. http://dx.doi.org/10.1016/j. eswa.2010.06.086

RAMANATHAN, R. Supplier selection problem: integrating DEA with the approaches of total cost of ownership and AHP. Supply Chain Management: An International Journal, v. 12, p. 258-261, 2007. http:// dx.doi.org/10.1108/13598540710759772

RAZMI, J.; RAFIEI, H. An integrated analytic network process with mixed-integer non-linear programming to supplier selection and order allocation. International Journal Advanced Manufacture Technology, v. 49 , p. $1195-1208,2010$. http://dx.doi.org/10.1007/ s00170-009-2445-5

RAZMI, J.; RAFIEI, H.; HASHEMI, M. Designing a decision support system to evaluate and select suppliers using fuzzy analytic network process. Computers \& Industrial Engineering, v. 57, p. 1282-1290, 2009. http://dx.doi.org/10.1016/j.cie.2009.06.008

REZAEI, J.; DAVOODI, M. A deterministic, multiitem inventory model with supplier selection and imperfect quality. Applied Mathematical Modelling, v. 32, p. 2106-2116, 2008. http://dx.doi.org/10.1016/j. apm.2007.07.009

RIBEIRO, J. R. Método para Solução do problema de seleção de fornecedores utilizando apoio multicritério à decisão. 2008. Dissertação (Mestrado em Engenharia Aeronaútica e Mecânica)-Instituto Tecnológico de Aeronáutica, São José dos Campos, 2008.

RUMELHART, D. E.; HINTON, G. E.; McCLELLAND, J. L. A General Framework for Parallel Distributed Processing. In: RUMELHART, D. E.; McCLELLAND, J. L. Parallel Distributed Processing: Explorations in the Microstructure of Cognition. v. 1. Cambridge: MIT Press, 1986.

SAATY, T. L. The Analytic Network Process. New York: McGraw Hill, 1980.

SAATY, T. L. Decision Making With Dependence And Feedback: The Analytic Network Process. Pittsburgh: RWS Publications, 1996.

SAEN, R. F. A new mathematical approach for suppliers selection: Accounting for non-homogeneity is important. Applied Mathematics and Computation, v. 185, p. 84-95, 2007a. http://dx.doi.org/10.1016/j. amc.2006.07.071

SAEN, R. F. Suppliers selection in the presence of both cardinal and ordinal data. European Journal of Operational Research, v. 183, p. 741-747, 2007b. http://dx.doi.org/10.1016/j.ejor.2006.10.022

SAEN, R. F. Restricting weights in supplier selection decisions in the presence of dual-role factors. Applied Mathematical Modelling, v. 34, p. 2820-2830, 2010. http://dx.doi.org/10.1016/j.apm.2009.12.016

SALMINEN, P.; HOKKANEN, J.; LAHDELMA, R. Comparing multicriteria methods in the context of environmental problems. European Journal of Operational Research, v. 104, p. 485-496, 1998.
SALOMON, V. A. P.; MARINS, F. A. S.; DUDUCH, M. A. Multiple-decisions decision-making applied to the supplier selection for assembly line equipments in an automotive industry. In: INTERNATIONAL SYMPOSIUM ON THE ANALYTIC HIERARCHY PROCESS, 9., 2007, Vina del Mar. Proceedings... Santiago: Fulcrum, 2007.

SAMPAIO, R. F.; MANCINI, M. C. Estudos de Revisão Sistemática: Um guia para síntese criteriosa da evidência científica. Revista brasileira de fisioterapia, v. 11, n. 1, p. 83-89, 2007. http://dx.doi.org/10.1590/ S1413-35552007000100013

SANAYEI, A. et al. An integrated group decision-making process for supplier selection and order allocation using multi-attribute utility theory and linear programming. Journal of the Franklin Institute, v. 345, p. 731-747, 2008. http://dx.doi.org/10.1016/j. jfranklin.2008.03.005

SANAYEI, A.; MOUSAVI, S. F.; YAZDANKHAH, A. Group decision making process for supplier selection with VIKOR under fuzzy environment. Expert Systems with Applications, v. 37, p. 24-30, 2010. http://dx.doi. org/10.1016/j.eswa.2009.04.063

SARKIS, J.; TALLURI, S. A model for strategic supplier selection. In: INTERNATIONAL IPSERA CONFERENCE, 9., 2000, London. Proceedings... London: Richard Ivey Business School, 2000. p. 652-661.

SAWIK, T. Supplier selection in make-to-order environment with risks. Mathematical and Computer Modelling, v. 53, p. 1670-1679, 2011. http://dx.doi.org/10.1016/j. mcm.2010.12.039

SEVKLI, M. et al. Hybrid analytical hierarchy process model for supplier selection. Industrial Management \& Data Systems, v. 108, p. 122-142, 2008. http://dx.doi. org/10.1108/02635570810844124

SHEMSHADI, A. et al. A fuzzy VIKOR method for supplier selection based on entropy measure for objective weighting. Expert Systems with Applications, v. 38, n. 10, 2011. http://dx.doi.org/10.1016/j.eswa.2011.03.027

SHEN, C.; YU, K. Enhancing the efficacy of supplier selection decision-making on the initial stage of new product development: A hybrid fuzzy approach considering the strategic and operational factors simultaneously. Expert Systems with Applications, v. 36, p. 11271-11281, 2009. http://dx.doi.org/10.1016/j. eswa.2009.02.083

SHU, M.; WU, H. Quality-based supplier selection and evaluation using fuzzy data. Computers \& Industrial Engineering, v. 57, p. 1072-1079, 2009. http://dx.doi. org/10.1016/j.cie.2009.04.012

SHYUR, H.; SHIH, H. A hybrid MCDM model for strategic vendor selection. Mathematical and Computer Modelling, v. 44, p. 749-761, 2006. http://dx.doi. org/10.1016/j.mcm.2005.04.018

SONGHORI, J. R.; KHAKBAZ, M. H. An integrated fuzzy group decision making/fuzzy linear programming (FGDMLP) framework for supplier evaluation and order allocation. International Journal Advanced Manufacture Technology, v. 43, p. 590-607, 2009. http://dx.doi.org/10.1007/s00170-008-1719-7

SONGHORI, M. J. et al. A supplier selection and order allocation model with multiple transportation alternatives. International Journal Advanced Manufacture 
Technology, v. 52, p. 365-376, 2011. http://dx.doi. org/10.1007/s00170-010-2697-0

STEUER, R. E. Multiple Criteria Optimization: Theory, Computations, and Application. New York: John Wiley \& Sons, Inc, 1986.

THRULOGACHANTAR, P.; ZAILANI, S. The influence of purchasing strategies on manufacturing performance. Journal of Manufacturing Technology Management, v. 22, p. 641-663, 2011. http://dx.doi. org/10.1108/17410381111134482

TING, S.; CHO, D. An integrated approach for supplier selection and purchasing decisions. Supply Chain Management: An International Journal, v. 13, p. 116-127, 2008. http://dx.doi. org/10.1108/13598540810860958

TSAI, Y. L.; YANG, Y. J.; LIN, C. A dynamic decision approach for supplier selection using ant colony system. Expert Systems with Applications, v. 37, p. 83138321, 2010. http://dx.doi.org/10.1016/j.eswa.2010.05.053

USTUN, O.; DERMITAS, E. A. An integrated multi-objective decision-making process for multi-period lot-sizing with supplier selection. Omega, v. 36, p. 509-521, 2008 a. http://dx.doi.org/10.1016/j.omega.2006.12.004

USTUN, O.; DERMITAS, E. A. Multi-period lot-sizing with supplier selection using achievement scalarizing functions. Computers \& Industrial Engineering, v. 54, p. 918-931, 2008b. http://dx.doi.org/10.1016/j. cie.2007.10.021

VADAHNI, B. et al. Extension of the ELECTRE method for decision-making problems with interval weights and data. International Journal Advanced Manufacture Technology, v. 50, p. 793-800, 2010. http://dx.doi. org/10.1007/s00170-010-2537-2

VANTEDDU, G.; CHINNAM, R.; GUSHIKIN, O. Supply chain focus dependent supplier selection problem. International Journal of Production Economics, v. 129, p. 204-216, 2011. http://dx.doi.org/10.1016/j. ijpe.2010.10.003

VINODH, S.; RAMIYA, R. A.; GAUTHAM, S. G. Application of fuzzy analytic network process for supplier selection in a manufacturing organisation. Expert Systems with Applications, v. 38, p. 272-280, 2011. http://dx.doi.org/10.1016/j.eswa.2010.06.057

WANG, J.; CHENG, C.; KUN-CHENG, H. Fuzzy hierarchical TOPSIS for supplier selection. Applied Soft Computing, v. 9, p. 377-386, 2009. http://dx.doi. org/10.1016/j.asoc.2008.04.014

WANG, T.; YANG, Y. A fuzzy model for supplier selection in quantity discount environments. Expert Systems with Applications, v. 36, p. 12179-12187, 2009. http:// dx.doi.org/10.1016/j.eswa.2009.03.018

WANG, W. A fuzzy linguistic computing approach to supplier evaluation. Applied Mathematical Modelling, v. 34, p. 3130-3141, 2010. http://dx.doi.org/10.1016/j. apm.2010.02.002

WEBER, C. A.; CURRENT, J. R.; BENTON, W. C. Vendor selection criteria and methods. European Journal of
Operational Research, v. 50, p. 2-18, 1991. http:// dx.doi.org/10.1016/0377-2217(91)90033-R

WU, D. Supplier selection: A hybrid model using DEA, decision tree and neural network. Expert Systems with Applications, v. 36, p. 9105-9112, 2009. http://dx.doi. org/10.1016/j.eswa.2008.12.039

WU, D. D. A systematic stochastic efficiency analysis model and application to international supplier performance evaluation. Expert Systems with Applications, v. 37, p. 6257-6264, 2010. http://dx.doi.org/10.1016/j. eswa.2010.02.097

WU, W. et al. An integrated multi-objective decision-making process for supplier selection with bundling problem. Expert Systems with Applications, v. 36, p. 2327 2337, 2009. http://dx.doi.org/10.1016/j.eswa.2007.12.022

XIA, W.; WU, Z. Supplier selection with multiple criteria in volume discount environments. Omega, v. 35, p. 494-504, 2007. http://dx.doi.org/10.1016/j. omega.2005.09.002

YANG, C.; CHEN, B. Supplier selection using combined analytical hierarchy process and grey relational analysis. Journal of Manufacturing Technology Management, v. 17, p. 926-941, 2006. http://dx.doi. org/10.1108/17410380610688241

YANG, J. L. et al. Vendor selection by integrated fuzzy MCDM techniques with independent and interdependent relationships. Information Sciences, v. 178, p. 41664183, 2008. http://dx.doi.org/10.1016/j.ins.2008.06.003

YÜCEL, A.; GÜNERI, A. F. A weighted additive fuzzy programming approach for multi-criteria supplier selection. Expert Systems with Applications, v. 38, p. 6281-6286, 2011. http://dx.doi.org/10.1016/j. eswa.2010.11.086

ZADEH, L. A. Fuzzy Sets. Information and Control, v. 8 , p. 338-353, 1965. http://dx.doi.org/10.1016/ S0019-9958(65)90241-X

ZEYDAN, M.; ÇOLPAN, C.; ÇOBANOGLU, C. A combined methodology for supplier selection and performance evaluation. Expert Systems with Applications, v. 38, p. 2741-2751, 2011. http://dx.doi. org/10.1016/j.eswa.2010.08.064

ZHANG, D. An novel approach to supplier selection based on vague sets group decision. Expert Systems with Applications, v. 36, p. 9557-9563, 2009. http://dx.doi. org/10.1016/j.eswa.2008.07.053

ZHANG, J.; ZHANG, M. Supplier selection and purchase problem with fixed cost and constrained. International Journal of Production Economics, v. 129, p. 1-7, 2011. http://dx.doi.org/10.1016/j.ijpe.2010.08.003

ZHAO, K.; YU, X. A case based reasoning approach on supplier selection in petroleum enterprises. Expert Systems with Applications, v. 38, p. 6839-6847, 2011. http://dx.doi.org/10.1016/j.eswa.2010.12.055

ZOLGHADRI, M. Power-based supplier selection in product development projects. Computers in Industry, v. 62, n. 5, 2011. http://dx.doi.org/10.1016/j. compind.2010.12.001 
